\title{
The Strongest Magnetic Fields on the Coolest Brown Dwarfs
}

\author{
Melodie M. Kao ${ }^{1,2}$ (iD), Gregg Hallinan ${ }^{2}$, J. Sebastian Pineda ${ }^{3}$ (D), David Stevenson ${ }^{4}$ (D), and Adam Burgasser ${ }^{5}$ (D) \\ ${ }^{1}$ California Institute of Technology, Department of Astronomy, 1200 E California Boulevard, MC 249-17, Pasadena, CA 91125, USA \\ ${ }^{2}$ Arizona State University, School of Earth and Space Exploration, 550 E Tyler Mall, PSF 686, Tempe, AZ 85287, USA; mkao@ asu.edu \\ ${ }^{3}$ University of Colorado Boulder, Laboratory for Atmospheric and Space Physics, 3665 Discovery Drive, Boulder CO 80303, USA \\ ${ }^{4}$ California Institute of Technology, Division of Geological \& Planetary Sciences, 1200 E California Boulevard, MC 150-21, Pasadena, CA 91125, USA \\ ${ }^{5}$ University of California San Diego, Center for Astrophysics and Space Sciences, 9500 Gilman Drive, MC 0424, La Jolla, CA 92093, USA \\ Received 2017 August 2; revised 2018 May 2; accepted 2018 May 3; published 2018 July 31
}

\begin{abstract}
We have used NSF's Karl G. Jansky Very Large Array to observe a sample of five known radio-emitting late-L and $\mathrm{T}$ dwarfs ranging in age from $\sim 0.2$ to $3.4 \mathrm{Gyr}$. We observed each target for seven hours, extending to higher frequencies than previously attempted and establishing proportionally higher limits on maximum surface magnetic field strengths. Detections of circularly polarized pulses at 8-12 GHz yield measurements of 3.2-4.1 kG localized magnetic fields on four of our targets, including the archetypal cloud variable and likely planetary-mass object T2.5 dwarf SIMP J01365663+0933473. We additionally detect a pulse at $15-16.5 \mathrm{GHz}$ for the T6.5 dwarf 2MASS 10475385 +2124234 , corresponding to a localized $5.6 \mathrm{kG}$ field strength. For the same object, we tentatively detect a $16.5-18 \mathrm{GHz}$ pulse, corresponding to a localized $6.2 \mathrm{kG}$ field strength. We measure rotation periods between 1.47-2.28 hr for 2MASS J10430758+2225236, 2MASS J12373919+6526148, and SDSS J04234858-0414035, supporting (i) an emerging consensus that rapid rotation may be important for producing strong dipole fields in convective dynamos, and/or (ii) rapid rotation is a key ingredient for driving the current systems powering auroral radio emission. We observe evidence of variable structure in the frequency-dependent time series of our targets on timescales shorter than a rotation period, suggesting a higher degree of variability in the current systems near the surfaces of brown dwarfs. Finally, we find that age, mass, and temperature together cannot account for the strong magnetic fields produced by our targets.
\end{abstract}

Key words: brown dwarfs - planets and satellites: aurorae - planets and satellites: magnetic fields - radio continuum: stars - stars: individual (2MASS 10430758+2225236, 2MASS 12373919+6526148, SDSS

04234858-0414035, SIMP J01365662+0933473) - stars: magnetic field

\section{Introduction}

Characterizing magnetic fields in the coolest dwarfs and eventually exoplanets can provide valuable insight into the formation, emission, and evolution of planets through stars. For instance, they are key players in disk accretion onto pre-mainsequence T Tauri stars (Hartmann et al. 2016), affecting planet formation mechanisms. Plasma flow across magnetic field lines drives large-scale currents in brown dwarf and planetary systems, producing auroral emission that likely contributes to the optical and infrared variability traditionally attributed to atmospheric clouds (e.g., Artigau et al. 2009; Radigan et al. 2014; Badman et al. 2015; Hallinan et al. 2015; Kao et al. 2016). Magnetic fields have been invoked to explain fundamental properties such as inflated radii in planets and stars (Batygin \& Stevenson 2010; Kervella et al. 2016). Finally, they can mitigate the erosion of planetary atmospheres from strong stellar winds and coronal mass ejections, a special concern for planets in the habitable zones of $\mathbf{M}$ dwarfs and young stars (Vidotto et al. 2013; Brain et al. 2015; Leblanc et al. 2015).

To characterize such magnetic fields, it is important to understand the physical principles driving field generation in fully convective objects, which remains an open question in dynamo theory. Applications of convective dynamos span a wide breadth of cases, including rocky planet inner cores, gas giant planets, brown dwarfs, and low-mass stars. Fully convective objects cannot rely on strong differential rotation occurring between radiative and convective zones to help drive their dynamos. However, they still exhibit magnetic activity like $\mathrm{H} \alpha, \mathrm{X}$-ray, and radio emission (e.g., Berger et al. 2001;
Burgasser et al. 2003; Berger et al. 2005; McLean et al. 2012; Schmidt et al. 2015; Pineda et al. 2016), and kilogauss fields have been confirmed for M, L, and T dwarfs (e.g., Hallinan et al. 2006, 2007, 2008; Reiners \& Basri 2007, 2009; Morin et al. 2010; Route \& Wolszczan 2012, 2016; Kao et al. 2016; Shulyak et al. 2017). Turbulence dissipates fossil fields within 10-100 years (Chabrier \& Küker 2006), implying that a dynamo must continuously regenerate these strong fields.

Efforts to elucidate magnetic behaviors of fully convective objects have included many fruitful investigations into the role of rotation. For instance, $\mathrm{H} \alpha$ and $\mathrm{X}$-ray emission are both tracers of hot chromospheres and coronae in F through mid-M stars heated in part by magnetic processes (Vernazza et al. 1981; Schmitt \& Rosso 1988; Ulmschneider 2003). Rotation appears to affect such magnetic processes, as $\mathrm{H} \alpha$ and X-ray emission scales with increasing surface rotation or decreasing Rossby $^{6}$ number Ro, which measures the effect of the Coriolis force in the inertial part of the fluid flow (the convective time derivative of velocity). At Ro $\sim 0.1$, the activity-rotation scaling appears to saturate at a constant $\log L_{\mathrm{X}, \mathrm{H} \alpha} / L_{\mathrm{bol}}$ (McLean et al. 2012), indicating a possible saturation of the influence of rotation on dynamo activity in mid-M and earliertype dwarfs. However, the neutral atmospheres of dwarfs $\gtrsim M 7$ may preclude magnetic heating processes of similar nature from occurring in the coolest brown dwarfs (Mohanty et al. 2002), underscoring the need for an alternative way to evaluate magnetism on the coolest brown dwarfs.

\footnotetext{
6 Quantified as Ro $\sim P / \tau_{c}$, where $P$ is the stellar rotation period and $\tau_{c}$ is the convective turnover time.
} 
Indeed, 邓M7 dwarfs exhibit systematically weaker $\mathrm{H} \alpha$ emission while $L_{\mathrm{X}} / L_{\mathrm{bol}}$ decreases with increasing $v \sin i$ or decreasing Ro (Mohanty \& Basri 2003; Reiners \& Basri 2008, 2010; Berger et al. 2010; McLean et al. 2012), and the Güdel-Benz relation appears to break down for objects later than M7 due to a suppression of X-ray luminosities, even when taking activity-rotation saturation into account (Berger et al. 2010; Williams et al. 2014). The precipitous drop-off of $\mathrm{X}$-ray emission from M7 and later objects indicates that such objects lack hot coronae. Consequently, previously established relationships between magnetic flux and tracers of coronal and chromospheric magnetic activity may not apply. This calls for comparisons of direct magnetic field measurements rather than observational proxies to rotation rates. Pulsing radio brown dwarfs in particular provide a rich probe of rotationally dependent magnetism, since their radio emission frequencies map to field strengths, while rotational modulation of the emission can provide rotation period measurements.

Models explore how different parameters quantifying competing forces such as Lorentz, buoyancy, and Coriolis affect energy exchange mechanisms at play in the magnetohydrodynamics occurring in fully convective dynamo regions. These models observe various dependencies between global magnetic field behaviors such as field topologies, magnetic energy, and time variation to observable object parameters such as luminosity, rotation, and age (e.g., Browning 2008; Christensen et al. 2009; Gastine et al. 2013; Yadav et al. 2016). Testing them requires a means to probe magnetism in the coolest objects: planets and brown dwarfs.

The unexpected detection of quiescent and flaring radio emission from the M9 brown dwarf LP 944-20 at 4.9 GHz and 8.5 GHz with NSF's Karl G. Jansky Very Large Array (VLA) at the beginning of this millennium heralded an unexpected new window into brown dwarf magnetism (Berger et al. 2001). This discovery paved the way to the subsequent detection of rotationally modulated and highly circularly polarized radio pulses attributed to the electron cyclotron maser (ECM) instability (Hallinan et al. 2006, 2007), which is the same process driving auroral radio emissions in the magnetized solar system planets (Zarka 1998).

The identification of auroral ECM emission from brown dwarfs was a crucial step to probing magnetic field strengths on the coolest brown dwarfs. For cool brown dwarfs with largely neutral atmospheres where collisions are negligible (the ratio of the plasma frequency to the electron cyclotron frequency is very low), emission occurs very near the electron cyclotron fundamental frequency $\nu_{\mathrm{MHz}} \sim 2.8 \times B_{\text {Gauss }}$ (Treumann 2006, and references therein). While auroral ECM emission cannot provide detailed insight into global magnetic field properties and its absence does not necessarily imply the absence of strong magnetic fields, detections provide powerfully direct measurements of field strengths at emitting regions within the magnetosphere.

In contrast, magnetic field measurements from the Zeeman broadening of magnetically sensitive spectral lines can return filling factor and surface-averaged field strengths with $\sim 15 \%$ 30\% uncertainties (Valenti et al. 1995; Johns-Krull \& Valenti 1996, 2000; Reiners \& Basri 2007; Shulyak et al. 2010; Reiners 2012). Zeeman Doppler imaging (ZDI) adds the ability to spatially distinguish different regions of different field strengths and reconstruct surface field topologies by fitting spectropolarimetric observations to those synthetically generated from test magnetic maps. Structure of opposite polarity on scales smaller than a spatial resolution element can cancel out, so ZDI is preferentially sensitive to the largest scales (Reiners \& Basri 2009; Yadav et al. 2015), with significant confusion between the dipole and quadrupole components, and $\sim 10 \%-30 \%$ uncertainties in dipole energies (Morin et al. 2010). Observations only probing some and not all of the Stokes parameters are further constrained in their abilities to fully capture complex field topologies (Rosén et al. 2015). Finally, known Landé factors remain limited and prevent Zeeman broadening and ZDI techniques from accessing L and later dwarfs (Berdyugina \& Solanki 2002; Shulyak et al. 2010).

Currently, the question of whether brown dwarf ECM emission is sensitive to large-scale (e.g. dipole) or small-scale fields remains unresolved However, a careful interpretation of the measurements allows for comparison to Zeeman broadening measurements and paves the way to extending observational tests of fully convective dynamos to the coolest brown dwarfs (Kao et al. 2016).

However, efficient detection of brown dwarf auroral radio emission eluded astronomers for over a decade, with an overall detection rate of just $\sim 10 \%$ in previous volume-limited surveys (Antonova et al. 2013; Route 2016). Moreover, only one detection out of $\sim 60$ L6 or later targets had been achieved before 2016 (Route \& Wolszczan 2012), seriously hindering the application of ECM emission to testing dynamos mechanisms in the mass and temperature gap between planets and stars. Yet, the unprecedented discovery of a T6.5 dwarf emitting at $\sim 4 \mathrm{GHz}$ demonstrated that such emission could indeed extend to objects probing the substellar-planetary boundary (Route \& Wolszczan 2012).

We previously developed and tested a selection strategy for identifying likely ECM-emitting brown dwarf candidates by making use of an emerging connection between ECM emission and possible tracers of aurora (Kao et al. 2016). We selected targets with known $\mathrm{H} \alpha$ emission and/or optical/infrared variability, leading to the detection of ECM emission in four out of five new L7-T6.5 brown dwarf pilot targets at 4-8 GHz, confirming $>2.5 \mathrm{kG}$ magnetic fields. A subsequent study confirmed detectable levels of $\mathrm{H} \alpha$ emission for all but one of these targets (e.g., Burgasser et al. 2003; Pineda et al. 2016).

The addition of this collection of radio brown dwarf magnetic field measurements to the single previous measurement from the T6.5 dwarf 2MASS 10475385+2124234 (Route \& Wolszczan 2012; Williams \& Berger 2015) provided strong observational evidence that very cold brown dwarfs can generate kilogauss fields, as well as a means for initial tests of dynamo theory at $\sim 1000 \mathrm{~K}$ temperatures. Comparisons of ECM-derived magnetic field measurements to Zeeman-based measurements tentatively suggested that dynamos operating in the coolest brown dwarfs may in fact produce fields that differ from values predicted by the luminosity-driven Christensen et al. (2009) model.

Higher frequency measurements of these objects can provide yet tighter constraints, motivating this work. Observations of ECM auroral emissions in the solar system planets demonstrate that the emission drops off sharply at a cutoff frequency corresponding to the strength of the field near the surface of the. The persistence of highly circularly polarized and pulsing emission in our targets throughout the previously observed 4-8 GHz bandwidth suggested that the emitting electrons were still traversing the magnetospheres of our targets toward increasing magnetic flux. A detection of a cutoff in the ECM 
emission would provide the tightest radio-derived constraints on brown dwarf magnetic fields, and in fact none has yet been detected in any brown dwarfs to date.

Finally, the rotational modulation of auroral ECM emission provides a means of measuring rotational periods and eventually testing dynamo models that examine the role of rotation by observing our known auroral radio emitters for longer time blocks to achieve full rotational phase coverage. Previous studies verified that pulse periods are consistent with rotational broadening from spectral lines (Berger et al. 2005, 2009; Hallinan et al. 2006, 2008).

In this work, we present new $8-12 \mathrm{GHz}$ and $12-18 \mathrm{GHz}$ observations of targets detected in our previous $4-8 \mathrm{GHz}$ pilot survey (Sections 3, 4.1). We carefully trace the evolution of auroral ECM pulses through 1 or $1.5 \mathrm{GHz}$ sub-bands (Section 4.2) and measure rotation periods (Section 4.3). Finally, we comment on implications for dynamo theory (Section 6).

\section{Targets}

Our sample of targets is discussed in Kao et al. (2016) but is again summarized here with updated literature for completeness. All targets are known to emit ECM emission at $4-8 \mathrm{GHz}$ (Kao et al. 2016).

2MASS $10475385+2124234.2 \mathrm{M} 1047$ is a T6.5 dwarf with known weak $\left[L_{\mathrm{H}_{\alpha}} / L_{\mathrm{bol}}\right] \sim-5.5$ (Burgasser et al. 2003) and was the first $\mathrm{T}$ dwarf detected at radio frequencies (Route \& Wolszczan 2012). The detected emission was highly circularly polarized $(\gtrsim 72 \%)$ at $4.75 \mathrm{GHz}$. Follow-up observations detected both quiescent and ECM emission up to $10 \mathrm{GHz}$ (Williams et al. 2013; Williams \& Berger 2015), the latter of which was used to measure a $\sim 1.77 \mathrm{hr}$ rotation period up through $10 \mathrm{GHz}$. We included 2M1047 in our pilot survey to examine long-term variability and detected both pulsed and quiescent emission through $8 \mathrm{GHz}$. Using $\mathrm{H}_{2} \mathrm{O}$ and $\mathrm{K} / \mathrm{H}$ indices, Kao et al. (2016) derived $T_{\text {eff }}=869_{-29}^{+35} \mathrm{~K},>0.026 M_{\odot}$ estimated mass, and $>2.5$ Gyr age.

SIMP J01365662+0933473. SIMP0136 is a T2.5 dwarf well known for periodic $(P=2.3895 \pm 0.0005 \mathrm{hr})$ and highamplitude $(>5 \%) \mathrm{J}-$ and $\mathrm{K}_{s}$-band photometric variability (Artigau et al. 2009; Croll et al. 2016). High-amplitude infrared variability appears to occur at a higher rate in $\mathrm{L} / \mathrm{T}$ transition dwarfs (Radigan 2014; Radigan et al. 2014) and has been attributed to the onset of patchy clouds (Ackerman \& Marley 2001; Burgasser et al. 2002b; Marley et al. 2010; Apai et al. 2013; Radigan et al. 2014) to explain wavelengthdependent variability. No $\mathrm{H} \alpha$ emission has been detected down to $\left[L_{\mathrm{H}_{\alpha}} / L_{\mathrm{bol}}\right]<-6.6$, but it has anomalously strong $\mathrm{Li}$ I at $\mathrm{EW}=6.6 \pm 1.0$ and $7.8 \pm 1.0 \AA$ for two different nights and is the latest-type object with a clear lithium detection, indicative of a young age (Pineda et al. 2016). Kao et al. (2016) derived $T_{\text {eff }}=1089_{-54}^{+62}, 0.022_{-0.012}^{+0.015} M_{\odot}$ estimated mass, and $0.6_{-0.3}^{+1.1}$ Gyr age. Recently, Gagné et al. (2017) reported that SIMP0136 may be a member of the $\sim 200$ Myr old Carina-Near moving group. Using an empirical measurement of its bolometric luminosity and the the Saumon \& Marley (2008) models, they inferred $R=1.22 \pm 0.01 R_{\mathrm{J}}$, which together predicted $T_{\text {eff }}=1098 \pm 6 \mathrm{~K}$ and $M=12.7 \pm 1.0 M_{\mathrm{J}}$. New $v \sin i$ measurements and its photometric periodicity further constrained $R>1.01 \pm 0.02 R_{\mathrm{J}}$ and $M<42.6_{-2.4}^{+2.5} M_{\mathrm{J}}$.

2MASS J10430758+2225236. $2 \mathrm{M} 1043$ is an unusually red L8 dwarf with previously reported tentative $\mathrm{H} \alpha$ emission (Cruz et al. 2007). Pineda et al. (2016) confirmed $\left[L_{\mathrm{H}_{\alpha}} / L_{\mathrm{bol}}\right]=$
$-5.8 \pm 0.2$ as well as a tentative Li I absorption line with $\mathrm{EW}=10 \pm 3 \AA$. Kao et al. (2016) derived $T_{\text {eff }}=1390 \pm$ $180 \mathrm{~K}, 0.011_{-0.005}^{+0.011} M_{\odot}$ estimated mass, and $0.6_{-0.3}^{+4.6} \mathrm{Gyr}$ age.

2MASS J12373919+6526148. 2M1237 is a T6.5 dwarf with anomalously hyperactive $\mathrm{H} \alpha$ emission at $\left[L_{\mathrm{H}_{\alpha}} / L_{\mathrm{bol}}\right] \sim-4.2$ (Burgasser et al. 2000, 2003) with conflicting evidence of Jband variability (Burgasser et al. 2002a; Artigau et al. 2003). Kao et al. (2016) derived $T_{\text {eff }}=831_{-27}^{+31} \mathrm{~K},>0.028 M_{\odot}$ estimated mass, and $>3.4 \mathrm{Gyr}$ age.

SDSS J04234858-0414035. SDSS0423 is an L6/T2 binary with 0." 16 separation (Burgasser et al. 2005; Carson et al. 2011) and strong $\mathrm{H} \alpha$ emission $(\mathrm{EW}=3 \AA$ ) and Li I absorption $(\mathrm{EW}=11 \AA$ ) (Kirkpatrick et al. 2008). Pineda et al. (2016) confirmed $\mathrm{H} \alpha \mathrm{EW}=2.95 \pm 0.3 \AA$ and $\mathrm{Li} \mathrm{I} \mathrm{EW}=11.1 \pm$ $0.4 \AA$. It additionally exhibits J- and K-band but no $I_{c}$ photometric variability (Enoch et al. 2003; Clarke et al. 2008; Wilson et al. 2014). Kao et al. (2016) derived $T_{\text {eff }}=$ $1678_{-137}^{+174} \mathrm{~K}, 0.015_{-0.006}^{+0.021} M_{\odot}$ estimated mass, and $0.49_{-0.17}^{+0.62} \mathrm{Gyr}$ age, although these values are uncertain given that they are based on blended light spectra.

\section{Observations}

We observed four of our sources with previous C-band $(4-8 \mathrm{GHz})$ detections at $\mathrm{X}$ band $(8-12 \mathrm{GHz})$ and one source (2M1047) that had a previous $\mathrm{X}$ band detection at $\mathrm{Ku}$ band $(12-18 \mathrm{GHz})$ with the full VLA. We used the WIDAR correlator in 3-bit observing mode for $4 \mathrm{GHz}$ or $6 \mathrm{GHz}$ bandwidth observations with $2 \mathrm{~s}$ integrations in $7 \mathrm{hr}$ time blocks for 35 total program hours. Observations took place during 2015 May in BnA configuration. Tables 1 and 2 summarize target properties and observations, respectively.

\subsection{Calibrations}

For SIMP0136 and SDSS0423, we calibrated our measurement sets using standard VLA flux calibrators 3C48 and $3 \mathrm{C} 147$, respectively, and nearby phase calibrators. Flux calibrators were observed at the beginning and end of each observing block and interpolated. After initially processing raw measurement sets with the VLA Calibration Pipeline, we manually flagged remaining radio frequency interference (RFI). Strong time-dependent RFI resulted in $\sim 71$ minutes of data loss near the end of the observing block for SDSS0423. Typical full-bandwidth sensitivity at BnA configuration for $7 \mathrm{hr}$ observing blocks ( $\sim 5.5 \mathrm{hr}$ and $\sim 4 \mathrm{hr}$ on source) is $1.2 \mu \mathrm{Jy}$ and $2.1 \mu \mathrm{Jy}$ for $\mathrm{X}$ and $\mathrm{Ku}$ bands, respectively. Typical 3-bit observations reach an absolute flux calibration accuracy of $\sim 5 \%$ by bootstrapping flux densities with standard VLA flux calibrators. To correct for flux errors resulting from gain phase variation over our observing window, we alternated between target and phase calibrator integrations, with 15-minute and 6-minute cycle times for $\mathrm{X}$ and $\mathrm{Ku}$ bands, respectively. Our gain solutions varied slowly and smoothly over time and without any ambiguous phase wraps, suggesting that this source of error is negligible.

For 2M1047, 2M1043, and 2M1237, we observed the flux calibrator 3C295, which is typically recommended only for low-frequency observations in compact configurations. This calibrator was fully resolved at both $\mathrm{X}$ and $\mathrm{Ku}$ bands for our observations. For targets observed at X bands (2M1043 and 2M1237), we modified the VLA scripted pipeline to use A configuration $8.464 \mathrm{GHz}$ and $11.064 \mathrm{GHz}$ model images 
Table 1

Survey Targets

\begin{tabular}{|c|c|c|c|c|c|c|c|c|}
\hline Object Name & $\begin{array}{l}\text { Abbrev. } \\
\text { Name }\end{array}$ & SpT & $\begin{array}{c}\text { Parallax } \\
\text { (mas) }\end{array}$ & $\begin{array}{l}\text { Distance } \\
(\mathrm{pc})\end{array}$ & $\begin{array}{c}\mu_{\alpha} \cos \delta \\
\left(\text { mas yr }^{-1}\right)\end{array}$ & $\begin{array}{c}\mu_{\delta} \\
\left.\text { (mas yr }^{-1}\right)\end{array}$ & Notes & References $^{\mathrm{a}}$ \\
\hline 2MASS $10475385+2124234$ & $2 \mathrm{M} 1047$ & T6.5 & $94.73 \pm 3.81$ & $10.56 \pm 0.52$ & $-1714 \pm 7$ & $-489 \pm 4$ & $\mathrm{H} \alpha$, detected prior & $12 \underline{3} 4-8$ \\
\hline SIMP J01365662+0933473 & SIMP0136 & $\mathrm{T} 2.5$ & $162.32 \pm 0.89$ & $6.139 \pm 0.037$ & $1222.70 \pm 0.78$ & $0.5 \pm 1.2$ & IR var, no $\mathrm{H} \alpha^{\mathrm{b}}$ & $10109-81112$ \\
\hline 2MASS J10430758+2225236 & $2 \mathrm{M} 1043$ & L8 & $\ldots$ & $16.4 \pm 3.2$ & $-134.7 \pm 11.6$ & $-5.7 \pm 17.0$ & $\mathrm{H} \alpha$ emission & 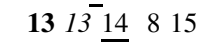 \\
\hline 2MASS J12373919+6526148 & 2M1237 & $\mathrm{T} 6.5$ & $96.07 \pm 4.78$ & $10.42 \pm 0.52$ & $-1002 \pm 8$ & $-525 \pm 6$ & $\mathrm{H} \alpha, \mathrm{IR} \operatorname{var}^{\mathrm{c}}$ & $116 \underline{3} \overline{4} 16-18$ \\
\hline SDSS J04234858-0414035 & SDSS0423 & $\mathrm{L} 7^{\mathrm{d}}$ & $65.93 \pm 1.7$ & $15.17 \pm 0.39$ & $-331 \pm 49$ & $76 \pm 11$ & $\mathrm{H} \alpha$, IR var, binary ${ }^{\mathrm{c}}$ & $\begin{array}{llll}19 & 320 & 8 & 21-28\end{array}$ \\
\hline
\end{tabular}

Notes.

- $\quad{ }^{\mathrm{a}}$ Citation legend: Discovery; $S p T$; Parallax, Distance, Proper Motion;

b $(8)$ reported upper limits $\left[L_{\mathrm{H}_{\alpha}} / L_{\mathrm{bol}}\right]<-6.6$.

c (16) and (18) report conflicting evidence of J-band variability.

d Secondary is spectral type T2.5 at orbital separation 0 " $16(26,27,28)$.

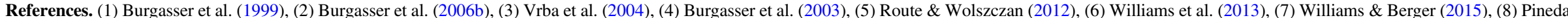

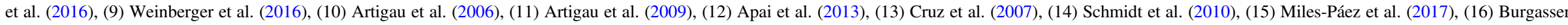

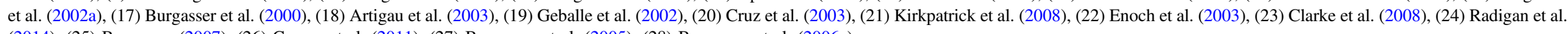
(2014), (25) Burgasser (2007), (26) Carson et al. (2011), (27) Burgasser et al. (2005), (28) Burgasser et al. (2006a). 
Table 2

Summary of Observations

\begin{tabular}{|c|c|c|c|c|c|c|c|c|c|c|}
\hline Object & $\begin{array}{l}\text { Band } \\
(\mathrm{GHz})\end{array}$ & $\begin{array}{l}\text { Obs. } \\
\text { Date } \\
(2015)\end{array}$ & $\begin{array}{l}\text { Obs. } \\
\text { Block } \\
\text { (h) }\end{array}$ & $\begin{array}{l}\text { Time on } \\
\text { Source } \\
(s)\end{array}$ & $\begin{array}{c}\text { VLA } \\
\text { Configuration }\end{array}$ & $\begin{array}{c}\text { Synthesized Beam } \\
\text { Dimensions } \\
(\operatorname{arcsec} \times \operatorname{arcsec})\end{array}$ & $\begin{array}{l}\mathrm{rms} \\
(\mu \mathrm{Jy})\end{array}$ & $\begin{array}{c}\text { Phase } \\
\text { Calibrator }\end{array}$ & $\begin{array}{c}\text { Flux } \\
\text { Calibrator }\end{array}$ & $\begin{array}{l}\text { Ref. Set } \\
\text { Frequency } \\
\quad(\mathrm{GHz})\end{array}$ \\
\hline $2 \mathrm{M} 1047$ & $12.0-18.0$ & $05 / 18$ & 7.0 & 20870 & $\mathrm{BnA}$ & $0 . \prime 62 \times 0 . .50$ & $1.7,1.8$ & $\mathrm{~J} 1051+2119$ & $3 \mathrm{C} 295$ & 14.064 \\
\hline SIMP0136 & $8.0-12.0$ & $05 / 17$ & 7.0 & 20870 & $\mathrm{BnA}$ & $0 . \prime 66 \times 0 . .37$ & $1.3,1.1$ & $\mathrm{~J} 0149+0555$ & $3 \mathrm{C} 48$ & $\cdots$ \\
\hline 2M1043 & $8.0-12.0$ & $05 / 20$ & 7.0 & 20612 & $\mathrm{BnA}$ & $0 . \prime 60 \times 0 . ! 33$ & $1.0,1.0$ & $\mathrm{~J} 1051+2119$ & $3 C 295$ & 11.064 \\
\hline 2M1237 & $8.0-12.0$ & $05 / 18$ & 7.0 & 21484 & $\mathrm{BnA}$ & $0 . \prime 69 \times 0 . .43$ & $1.0,1.1$ & $\mathrm{~J} 1339+6328$ & $3 \mathrm{C} 295$ & 8.464 \\
\hline SDSS0423 & $8.0-12.0$ & $05 / 30$ & 7.0 & 17234 & $\mathrm{BnA}$ & $0 . \prime 68 \times 0 . .37$ & $1.2,1.4$ & J0423-0120 & $3 \mathrm{C} 147$ & $\cdots$ \\
\hline
\end{tabular}

observed on 2016 February 16 by VLA staff to set flux levels and determine bandpass solutions. The emission from 3C295 is stable within 1\% over 24-28 years for X and Ku bands (Perley \& Butler 2013). Because the lobed structure of 3C295 is resolved at our observing frequencies and the VLA sky sensitivity fringes are wavelength dependent, we expect there to be a discrepancy in flux densities bootstrapped using these different images of 3C295. To estimate the additional uncertainty in flux densities introduced by calibrating with 3C295, we compared the flux densities of each target's phase calibrator as bootstrapped by the different model images of 3C295. We list these flux densities in Table 3. These comparisons suggest that the flux densities of $2 \mathrm{M} 1043$ and $2 \mathrm{M} 1237$ have an additional $\sim 1 \%-7 \%$ uncertainty. We repeated the same process for our Ku band target (2M1047), but instead used model images of $3 \mathrm{C} 295$ at $14.064 \mathrm{GHz}$ and $16.564 \mathrm{GHz}$, which we expect to introduce an additional $\sim 8 \%$ uncertainty.

We flagged all data from 12 to $12.8 \mathrm{GHz}$ during the first $\sim 34$ minutes of our target observing scans for 2M1047 due to strong RFI. After manually flagging remaining RFI, we averaged all of the measurement sets down in time from $2 \mathrm{~s}$ integrations to $10 \mathrm{~s}$ for faster processing.

\subsection{Source Motion}

We corrected the 2MASS coordinates (Skrutskie et al. 2006) of our targets using the proper motion measurements listed in Table 1 to obtain expected source positions. For the known binary SDSS0423, we did not correct for orbital motion because its 0 ". 16 orbital separation is well within the synthesized beam resolution.

\section{Methods}

In this section, we describe our general approach to analyzing the data. In Section 5, we detail specific challenges encountered in the analysis of data for each target.

\subsection{Imaging}

We produced Stokes $I$ and Stokes $V$ images of each object (total and circularly polarized flux densities, respectively) with the Common Astronomy Software Applications (CASA) clean routine, modeling the sky emission frequency dependence with one term and using natural weighting. Pixel sizes were 0 " $04 \times 0$ " 04 . We searched for a point source at the proper motion-corrected coordinates of each target. For our targets calibrated with 3C295, we selected a single calibrated measurement set as a reference set, noted in Table 4 . We performed all subsequent reduction and analysis on this reference set.
Table 3

Comparison of Phase Calibrator Flux Densities

\begin{tabular}{lcccc}
\hline \hline Object & $\begin{array}{c}\text { Ref. Freq } \\
8.464 \mathrm{GHz} \\
(\mathrm{mJy})\end{array}$ & $\begin{array}{c}\text { Ref. Freq } \\
11.064 \mathrm{GHz} \\
(\mathrm{mJy})\end{array}$ & $\begin{array}{c}\text { Ref. Freq } \\
14.064 \mathrm{GHz} \\
(\mathrm{mJy})\end{array}$ & $\begin{array}{c}\text { Ref. Freq } \\
16.564 \mathrm{GHz} \\
(\mathrm{mJy})\end{array}$ \\
\hline 2M1047 & $\cdots$ & $\cdots$ & $603.7 \pm 0.4$ & $561.1 \pm 0.2$ \\
$2 \mathrm{M} 1043$ & $466.4 \pm 1.2$ & $469.0 \pm 1.3$ & $\cdots$ & $\cdots$ \\
$2 \mathrm{M} 1237$ & $173.3 \pm 1.0$ & $185.0 \pm 1.0$ & $\cdots$ & $\cdots$ \\
\hline
\end{tabular}

Flux densities and source positions were determined by fitting an elliptical Gaussian point source to the cleaned image of each object at its predicted coordinates using the CASA task imfit.

\subsection{Time Series: Detecting ECM Pulses}

We used the clean routine to model all sources within a primary beam of our targets and subtracted these sources from the UV visibility data using the CASA uvsub routine to prevent sidelobe contamination in our targets' time series. We then added phase delays to our visibility data using the CASA fixvis routine to place our targets at the phase center.

We checked all targets for highly circularly polarized flux density pulses to confirm the presence of ECM emission. Rather than searching for pulsed emission in Stokes $I$ and $V$, we elected to search for pulses in the rr and 11 correlations (rightand left-circularly polarized, respectively), where the signal-tonoise ratio $(\mathrm{S} / \mathrm{N})$ is a factor of $\sqrt{2}$ higher in cases where the pulsed emission is $100 \%$ circularly polarized, as is expected in an ideal case of ECM emission.

Using the CASA plotting routine plotms to export the real UV visibilities averaged across all baselines, channels, and spectral windows of the rr and 11 correlations at $10 \mathrm{~s}, 60 \mathrm{~s}$, and $120 \mathrm{~s}$ time resolutions, we created $\mathrm{rr}$ and $1 \mathrm{l}$ time series for all X-band targets at $8-9 \mathrm{GHz}, 9-10 \mathrm{GHz}, 10-11 \mathrm{GHz}$, $11-12 \mathrm{GHz}, 8-10 \mathrm{GHz}, 10-12 \mathrm{GHz}$, and $8-12 \mathrm{GHz}$ bandwidths to check for frequency-dependent ECM emission cutoff. We repeated the same procedure for $2 \mathrm{M} 1047$, but divided the total bandwidth into $12-13.5 \mathrm{GHz}, 13.5-15 \mathrm{GHz}$, $15-16.5 \mathrm{GHz}, \quad 16.5-18 \mathrm{GHz}, 12-15 \mathrm{GHz}, 15-18 \mathrm{GHz}$, and $12-18 \mathrm{GHz}$. Figures 1-3 show the time series for each object.

We identify pulses using the following method: we smooth each time series with a locally weighted first-degree polynomial regression and a smoothing window of $2.5 \%$ of the on-target time to prevent anomalous noise spikes, typically very narrow with $\sim$ single time resolution element widths, from erroneously being identified as a pulse while also preventing the smearing 

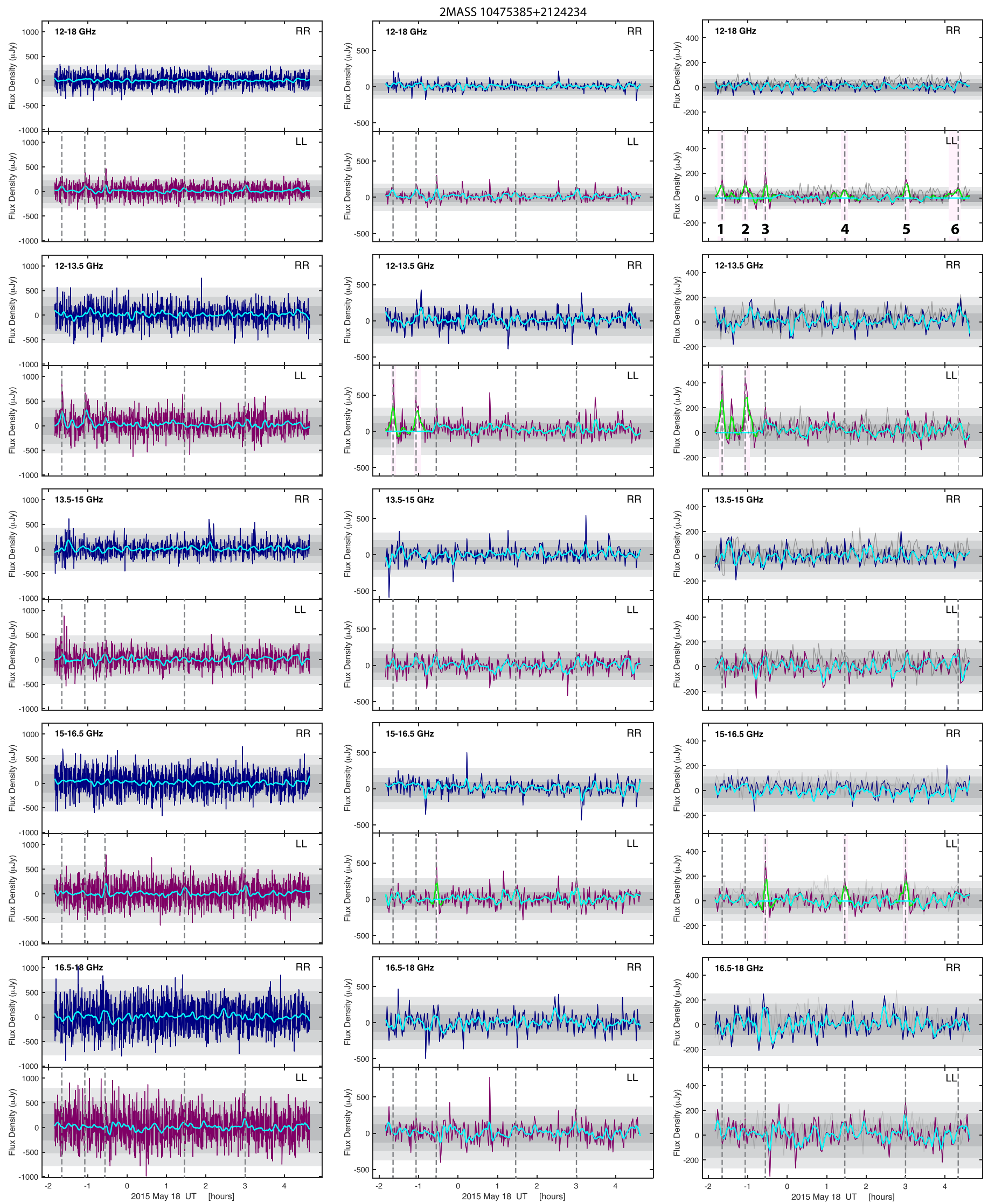

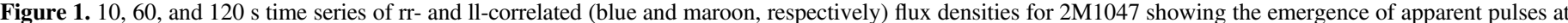

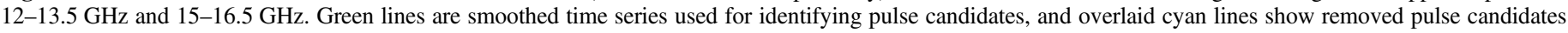

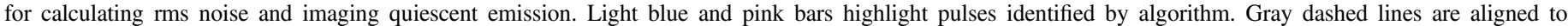

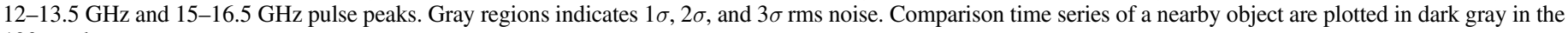
120 s column. 

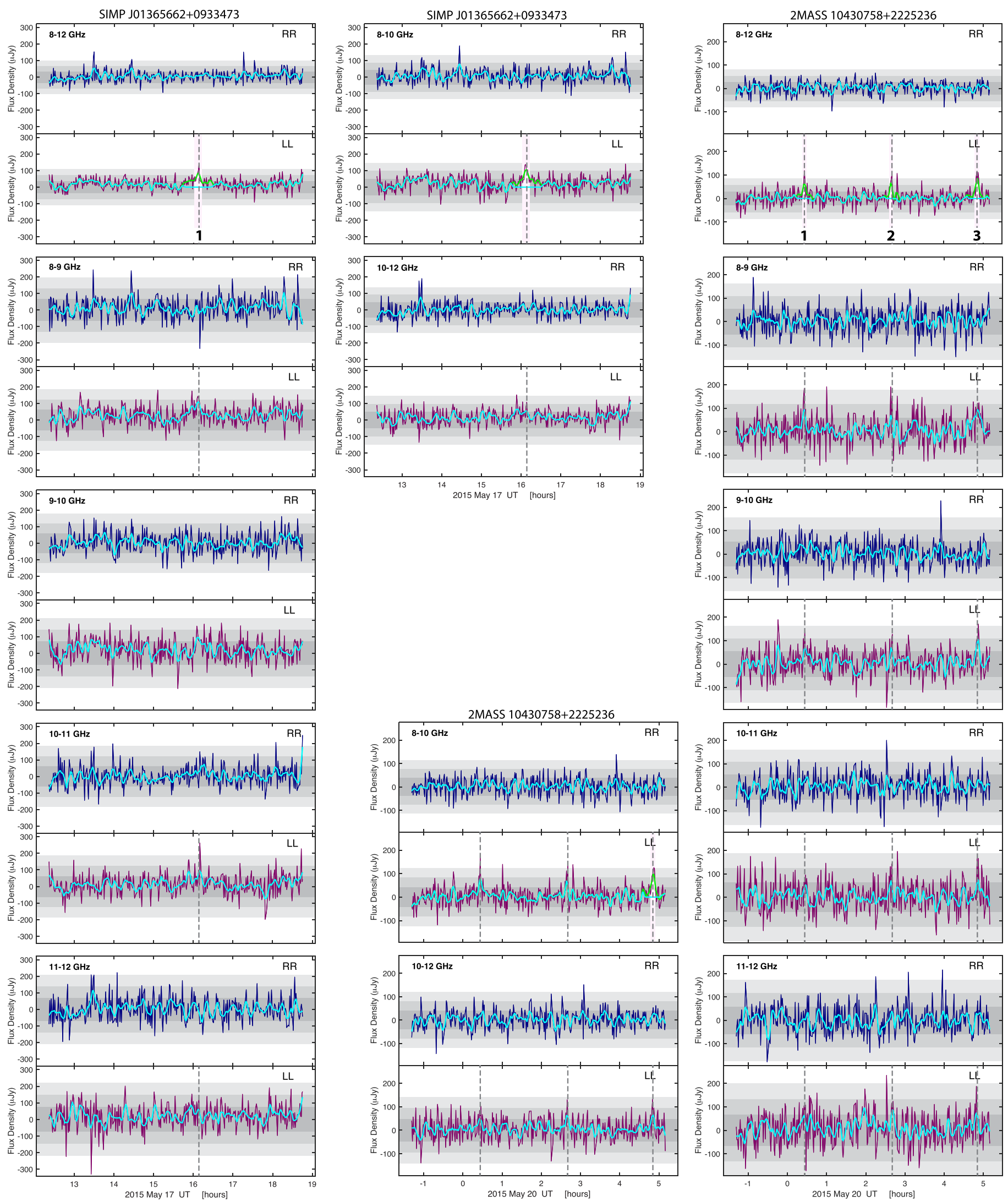

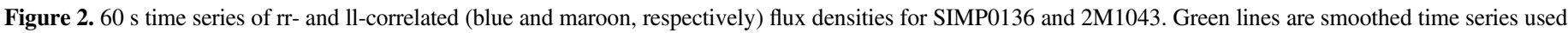

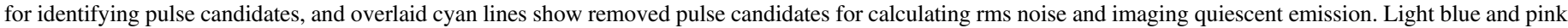
bars highlight pulses identified by algorithm. Gray dashed lines are aligned to pulse peaks. Gray regions indicates $1 \sigma, 2 \sigma$, and $3 \sigma$ rms noise. 

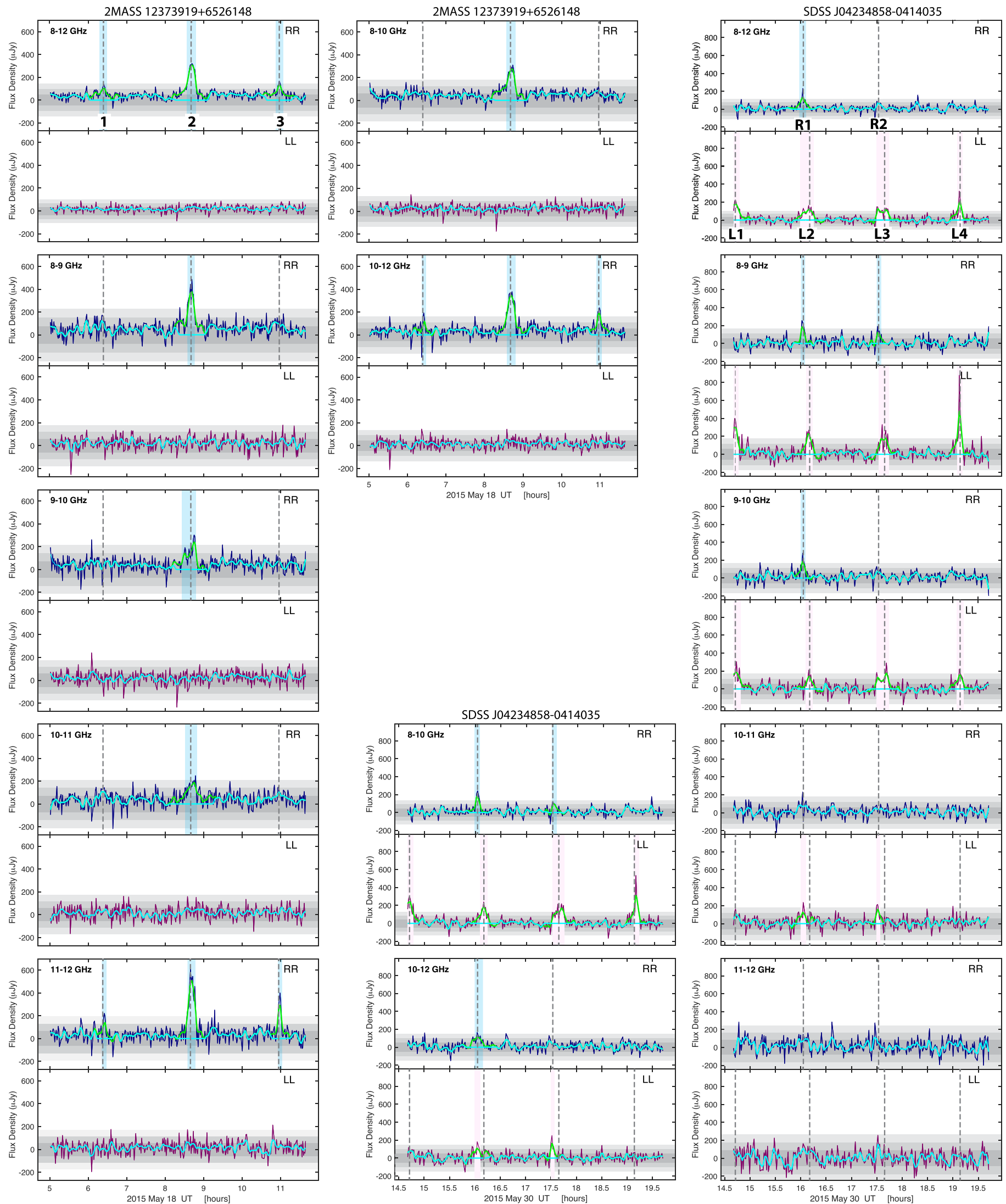

Figure 3. $60 \mathrm{~s} \mathrm{time} \mathrm{series} \mathrm{of} \mathrm{rr-} \mathrm{and} \mathrm{1l-correlated} \mathrm{(blue} \mathrm{and} \mathrm{maroon,} \mathrm{respectively)} \mathrm{flux} \mathrm{densities} \mathrm{for} \mathrm{2M1237} \mathrm{and} \mathrm{SDSS0423.} \mathrm{Green} \mathrm{lines} \mathrm{are} \mathrm{smoothed} \mathrm{time} \mathrm{series} \mathrm{used}$ for identifying pulse candidates, and overlaid cyan lines show removed pulse candidates for calculating rms noise and imaging quiescent emission. Light blue and pink bars highlight pulses identified by algorithm. Gray dashed lines are aligned to pulse peaks. Gray regions indicates $1 \sigma, 2 \sigma$, and $3 \sigma$ rms noise. 
Table 4

Summary of Initial Imaging Detections

\begin{tabular}{|c|c|c|c|c|c|}
\hline Object & $\begin{array}{c}\text { R.A. } \\
\text { (hh mm ss.ss) }\end{array}$ & $\begin{array}{c}\text { Decl. } \\
\text { (dd mm ss.ss) }\end{array}$ & $\begin{array}{c}\text { Stokes } I \\
(\mu \mathrm{Jy})\end{array}$ & $\begin{array}{c}\text { Stokes } V \\
(\mu \mathrm{Jy})\end{array}$ & $\begin{array}{l}\mathrm{S} / \mathrm{N} \\
(I, V)\end{array}$ \\
\hline 2M1047 & 104751.78 & +212414.90 & $21.9 \pm 1.3$ & $3.9 \pm 1.5$ & $16.8,2.6$ \\
\hline SIMP0136 & 013657.86 & +093347.00 & $85.7 \pm 1.3$ & $-23.8 \pm 1.1$ & $65.9,21.6$ \\
\hline 2M1043 & 104307.44 & +222523.31 & $9.5 \pm 1.0$ & $-4.7 \pm 1.0$ & $9.5,4.7$ \\
\hline 2M1237 & 123736.58 & +652605.70 & $35.0 \pm 1.0$ & $16.9 \pm 1.2$ & $35.0,14.1$ \\
\hline SDSS0423 & 042348.23 & -041402.15 & $15.4 \pm 1.2$ & $-0.5 \pm 1.4$ & $12.8,0.4$ \\
\hline
\end{tabular}

out of slightly wider legitimate pulses. We then identify $2 \sigma_{\text {rms }}$ outlier peaks in the smoothed time series and measure the FWHM of the smoothed pulse, where we use the rms of the time series as a proxy for any quiescent emission. In reality, these peaks lie above twice the quiescent emission, since the rms includes the peaks. Approximating each pulse as Gaussian, we define the full width of each pulse as three times the FWHM and remove each pulse from the raw time series. These initial steps remove the strongest pulses present in the time series that may cause weaker pulses from being automatically identified. Finally, we repeat the process once more to identify any other pulse candidates. Because sensitivity can be a concern at narrow time resolutions and bandwidths in the time series, we elected to conservatively set the detection threshold for this second iteration at $2 \sigma_{\mathrm{rms}}$ and separately verified the pulses by imaging each candidate pulse in Stokes $I$ and $V$ and comparing flux densities with that of the non-pulsed (quiescent) emission.

We confirm pulses with Stokes $I$ and $V$ imaging over the $60 \mathrm{~s}$ FWHM of each candidate pulse and measuring integrated Stokes $I$ and Stokes $V$ flux densities using the CASA routine imfit. In an initial set of fits, we allow the peak location to float and fix the semimajor and semiminor axes to the dimensions of a synthesized beam. Our fitting region is a $100 \times 100$ pixel region centered at the target location measured in Section 4.1. We select the highest $\mathrm{S} / \mathrm{N}$ pulse as a benchmark and perform a second iteration of fits while also holding the benchmark peak location constant. We list measurements for pulses with unambiguous imaging and rms noise limits for frequency sub-bands with no detection. Imaging for some sub-bands shows evidence for a possible point source at the expected target location that is not clearly distinguishable by eye from the noise in the image. We classify flux density measurements for these sub-bands as tentative detections and bootstrap the significance of the possible point source by randomly drawing 10,000 pointings in a $4096 \times 4096$ pixel $(2 ! 7 \times 2 ! 7)$ image and measuring the flux densities for a point source centered on these pointings.

We calculate the highest-likelihood percent circular polarization, where negative and positive percentages correspond to left- and right-circular polarizations, respectively. We report uncertainties that correspond to the upper and lower limits of the $68.27 \%$ confidence interval and record the evolution of pulse flux densities across sub-bands in Table 5 (2M1237), Table 6 (2M1047), Table 7 (SIMP0136 \& 2M1043), and Table 8 (SDSS0423). Some pulses appear to have Stokes $V$ fluxes that are higher than the Stokes $I$ fluxes, which is not physically possible. However, these anomalous excess flux densities are within the rms noise. For objects with $100 \%$ circular polarization, we give the lower-bounds of the $68.27 \%$ and $99.73 \%$ confidence intervals on the circular polarization.

We additionally measure quiescent emission by removing the full width of each pulse across the entire 4 or $6 \mathrm{GHz}$ bandwidth from our data and imaging the remaining emission, shown in Figure 4. We report the characteristics of the pulsed and quiescent emission in Tables 5-8.

\subsection{Measuring Rotation Periods}

Our data are well sampled with respect to pulse widths but very noisy and may contain low-amplitude or wide duty cycle peaks. Previous attempts have benefited from fitting the time series of relatively bright $\sim \mathrm{mJy}$ pulses (Hallinan et al. 2007, 2008; Williams \& Berger 2015; Route \& Wolszczan 2016), an order of magnitude brighter than the pulses in our targets. In contrast, for our data, some pulses do not become apparent until the data have been averaged to $60 \mathrm{~s}$ or $120 \mathrm{~s}$ resolutions, further introducing uncertainty when attempting to accurately identify pulses and their arrival times. For these reasons, we elected not to pursue a Levenberg-Marquardt or Monte Carlo time-of-arrival fitting (Williams \& Berger 2015; Route \& Wolszczan 2016) and instead employed three independent algorithms widely used in exoplanet transit and radial velocity searches. Using these algorithms has the added benefit of independently verifying the pulses that we identified in Section 4.2. The first is the classic Lomb-Scargle $(\mathrm{L}-\mathrm{S})$ periodogram, which relies on decomposing time series into Fourier components and is optimized to identify sinusoidally shaped periodic signals in time-series data, making this algorithm most appropriate for testing periodicity in broader pulses such as those observed in the SDSS0423 and SIMP0136 time series or even our targets' quiescent emission. The second method is the Plavchan periodogram, a brute-force method that derives periodicities in a method similar to that employed by phase dispersion minimization (Stellingwerf 1978), but circumvents period aliasing because it is binless (Plavchan et al. 2008; Parks et al. 2014). The Plavchan algorithm is not dependent on pulse shape and thus is sensitive to both sinusoiddominated variability and other pulse profiles. Finally, the shapes of some of the pulses bear resemblance to inverse light curves of planet transits, for which the box-fitting least-squares (BLS) algorithm is optimized (Kovács et al. 2002).

We generate periodograms for all of our objects using the $10 \mathrm{~s}$ time-averaged time series for the full-bandwidth data and at all sub-bands using the MATLAB L-S function plomb and the NASA Exoplanet Archive Periodogram Service ${ }^{7}$ for Plavchan and BLS periodograms. The Plavchan algorithm depends on two input parameters: number of outliers and

\footnotetext{
https://exoplanetarchive.ipac.caltech.edu/cgi-bin/Pgram/nph-pgram
} 
Table 5

2M1237: Pulsed and Quiescent Emission

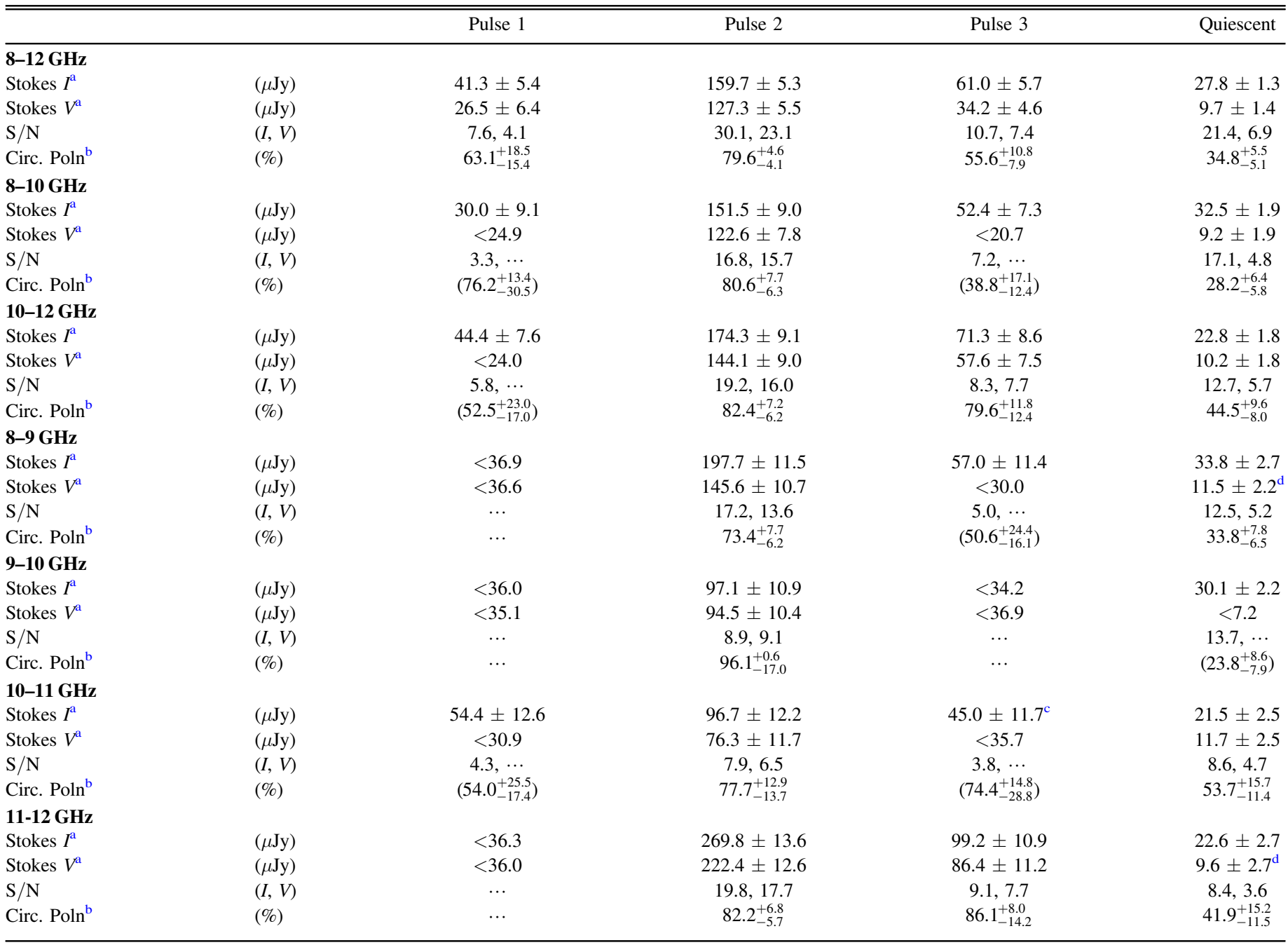

Notes.

${ }^{a}$ Reported flux densities are integrated over the FWHM of the full-bandwidth $60 \mathrm{~s}$ resolution data. Fixing fit parameters can result in overestimated uncertainties on the integrated and peak flux densities, so we report the rms image noise as the uncertainty $\sigma_{\mathrm{rms}}$. For targets with a clear visual non-detection, we list $3 \sigma_{\text {rms }}$.

${ }^{\mathrm{b}}$ Reported polarization fractions are highest-likelihood values, given the measured Stokes $I$ and Stokes $V$ flux densities. Uncertainties reflect upper and lower bounds of $68.27 \%$ confidence intervals. Negative and positive values indicate left- and right- circular polarizations, respectively. Lower-bound $68.27 \%$ and $99.73 \%$ confidence intervals are given for sub-bands with $100 \%$ circular polarization. Upper bounds are given in parentheses for objects without detectable levels of Stokes $V$ emission, assuming a $3 \sigma_{\mathrm{rms}}$ flux density and right circular polarization.

${ }^{\mathrm{c}}$ Tentative image detection (no clearly visually distinguishable $(I, V)$ point source). Bootstrapped significance is $99.20 \%$.

d Tentative image detection (no clearly visually distinguishable Stokes $V$ point source). Possible Stokes $I$ point sources are apparent at the expected location of 2M1237 but are not clearly distinguishable by eye from the noise in the image. Bootstrapped significance is $99.93 \%$ (8-9 GHz) and $99.39 \%(11-12 \mathrm{GHz})$.

fractional phase smoothing width, which we vary between $10 \%-30 \%$ of total data points and $0.025-0.1$, respectively. BLS depends on three input parameters: number of points per bin, minimum fractional period coverage by pulse, and maximum fraction period coverage. For BLS, we hold the minimum fractional period coverage constant at 0.01 , and we vary the number of points per bin and maximum fractional period coverage between $10-100$ and $0.1-0.3$, respectively. In most cases, the recovered periodicities do not depend significantly on these parameters and we discuss exceptions in Section 5.

We compare peaks with a false-alarm probability lower than $10 \%$ returned by the the $\mathrm{L}-\mathrm{S}$ algorithm to the most significant periods returned by the other algorithms in Figure 5 and visually inspect periods by phase-folding the time series in Figure 6 with the most significant period returned by each algorithm. We estimate uncertainties as the inverse of the FWHM of the frequency power peaks. We list periods returned by each algorithm in Table 9 and adopt the periods that result in the folded time series with the most visual agreement in pulse overlaps.

\section{Results}

2MASS J12373919+6526148. We detect 2M1237 in initial Stokes $I$ and Stokes $V$ imaging with an $\mathrm{S} / \mathrm{N}$ of 35.0 and 14.1, respectively. Table 4 gives the measured mean flux density and rms noise. These strong detections are due to weakly circularly 
Table 6

2M1047: Pulsed and Quiescent Emission

\begin{tabular}{|c|c|c|c|c|c|c|c|c|c|}
\hline & & Pulse 1 & Pulse 2 & Pulse 3 & Pulse 4 & Pulse 5 & Pulse 6 & Pulses 3-5 & $\overline{\text { Quiescent }}$ \\
\hline \multicolumn{10}{|l|}{ 12-18 GHz } \\
\hline Stokes $I^{\mathrm{a}}$ & $(\mu \mathrm{Jy})$ & $47.0 \pm 14.8^{\mathrm{c}}$ & $50.7 \pm 13.3$ & $63.8 \pm 12.9$ & $<46.8$ & $71 \pm 11.6$ & $31.0 \pm 7.0$ & $54.0 \pm 7.1$ & $7.4 \pm 2.2^{\mathrm{e}}$ \\
\hline Stokes $V^{\mathrm{a}}$ & $(\mu \mathrm{Jy})$ & $-46.4 \pm 14.3^{\mathrm{c}}$ & $<36.3$ & $<44.7$ & $<50.1$ & $-56 \pm 10.6$ & $<19.5$ & $-33.3 \pm 8.3$ & $<5.4$ \\
\hline $\mathrm{S} / \mathrm{N}$ & $(I, V)$ & $3.2,3.2$ & $3.8, \cdots$ & $4.9, \cdots$ & $\cdots$ & $6.1,5.3$ & $4.4, \cdots$ & $7.6,4.0$ & $3.4, \cdots$ \\
\hline Circ. Poln ${ }^{b}$ & $(\%)$ & $-90.2_{-2.0}^{+38.3}$ & $\left(-67.1_{-20.0}^{+24.2}\right)$ & $\left(-67.3_{-19.4}^{+24.0}\right)$ & $\cdots$ & $-76.8_{-13.8}^{+16.8}$ & $\left(-59.9_{-23.4}^{+20.1}\right)$ & $-60.6_{-39.0}^{+45.4}$ & $\cdots$ \\
\hline \multicolumn{10}{|l|}{ 12-13.5 GHz } \\
\hline Stokes $I^{\mathrm{a}}$ & $(\mu \mathrm{Jy})$ & $<91.5^{\mathrm{c}}$ & $143.4 \pm 17.6$ & $<84.6$ & $<78.9$ & $<81.0$ & $<38.4$ & $<48.3$ & $20.4 \pm 4.1^{\mathrm{e}}$ \\
\hline Stokes $V^{\mathrm{a}}$ & $(\mu \mathrm{Jy})$ & $-129.6 \pm 24.6^{\mathrm{c}}$ & $-78.9 \pm 21.7$ & $<81.3$ & $<77.7$ & $<78.6$ & $<38.3$ & $<45.6$ & $<12.3$ \\
\hline $\mathrm{S} / \mathrm{N}$ & $(I, V)$ & $\cdots, 5.3$ & $8.1,3.6$ & $\cdots$ & $\cdots$ & $\cdots$ & $\cdots$ & $\cdots$ & $5.0, \cdots$ \\
\hline Circ. Poln ${ }^{\mathrm{b}}$ & $(\%)$ & $(-72.8,-38.0)$ & $-54.2_{-18.8}^{+14.6}$ & $\cdots$ & $\cdots$ & $\cdots$ & $\cdots$ & $\cdots$ & $\cdots$ \\
\hline \multicolumn{10}{|l|}{ 13.5-15 GHz } \\
\hline Stokes $I^{\mathrm{a}}$ & $(\mu \mathrm{Jy})$ & $<105.0$ & $<71.7$ & $<80.4$ & $<72.0$ & $<72.3$ & $<41.7$ & $<44.1$ & $<10.5$ \\
\hline Stokes $V^{\mathrm{a}}$ & $(\mu \mathrm{Jy})$ & $<110.4$ & $<68.4$ & $<81.6$ & $<75.9$ & $<71.1$ & $<40.8$ & $<43.5$ & $<11.1$ \\
\hline $\mathrm{S} / \mathrm{N}$ & $(I, V)$ & $\cdots$ & $\cdots$ & $\cdots$ & $\cdots$ & $\cdots$ & $\cdots$ & $\cdots$ & $\cdots$ \\
\hline Circ. Poln ${ }^{b}$ & $(\%)$ & $\cdots$ & $\cdots$ & $\cdots$ & $\cdots$ & $\cdots$ & $\cdots$ & $\cdots$ & $\cdots$ \\
\hline \multicolumn{10}{|l|}{ 15-16.5 GHz } \\
\hline Stokes $I^{\mathrm{a}}$ & $(\mu \mathrm{Jy})$ & $<77.4$ & $<66.3$ & $125.4 \pm 25.8$ & $93.3 \pm 19.9$ & $93.7 \pm 24.0$ & $<38.7$ & $\begin{array}{c}1(I, V) \\
05.2 \pm 13.7\end{array}$ & $<12.3$ \\
\hline Stokes $V^{\mathrm{a}}$ & $(\mu \mathrm{Jy})$ & $<77.9$ & $<67.8$ & $<84.6$ & $<69.6$ & $<63.9$ & $<41.1$ & $-46.7 \pm 12.8$ & $<12.0$ \\
\hline $\mathrm{S} / \mathrm{N}$ & $(I, V)$ & $\cdots$ & $\cdots$ & $4.9, \cdots$ & $9.4, \cdots$ & $3.9, \cdots$ & $\cdots$ & $7.7,3.6$ & $\cdots$ \\
\hline Circ. Poln ${ }^{\mathrm{b}}$ & $(\%)$ & $\cdots$ & $\cdots$ & $\left(-64.8_{-20.8}^{+19.2}\right)$ & $\left(-71.4_{-16.8}^{+26.5}\right)$ & $\left(-64.1_{-21.8}^{+22.4}\right)$ & $\cdots$ & $-43.6_{-50.9}^{+35.7}$ & $\cdots$ \\
\hline \multicolumn{10}{|c|}{ 16.5-18 GHz } \\
\hline Stokes $I^{\mathrm{a}}$ & $(\mu \mathrm{Jy})$ & $<99.3$ & $<90.3$ & $<102.9$ & $<91.2$ & $91.5 \pm 28.7^{d}$ & $<54.0$ & $<57.3$ & $<15.6$ \\
\hline Stokes $V^{\mathrm{a}}$ & $(\mu \mathrm{Jy})$ & $<108.6$ & $<88.8$ & $<95.1$ & $<99.9$ & $-94.9 \pm 24.9^{\mathrm{d}}$ & $<52.8$ & $<56.4$ & $<15.6$ \\
\hline $\mathrm{S} / \mathrm{N}$ & $(I, V)$ & $\cdots$ & $\cdots$ & $\cdots$ & $\cdots$ & $3.2,3.8$ & $\cdots$ & $\cdots$ & $\cdots$ \\
\hline Circ. Poln ${ }^{b}$ & $(\%)$ & $\cdots$ & $\cdots$ & $\cdots$ & $\cdots$ & $-58.0,-14.3$ & $\cdots$ & $\cdots$ & $\cdots$ \\
\hline
\end{tabular}

Notes.

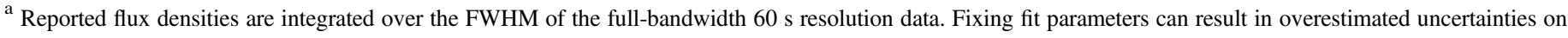
the integrated and peak flux densities, so we report the rms image noise as the uncertainty $\sigma_{\text {rms }}$. For targets with a clear visual non-detection, we list $3 \sigma_{\text {rms }}$.

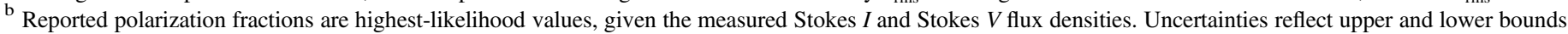

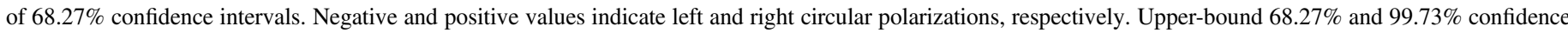

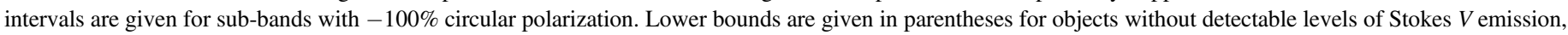
assuming a $3 \sigma_{\text {rms }}$ flux density and left circular polarization.

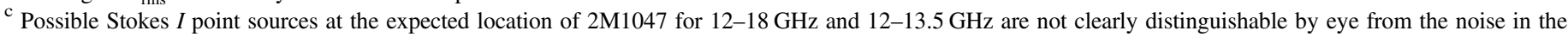

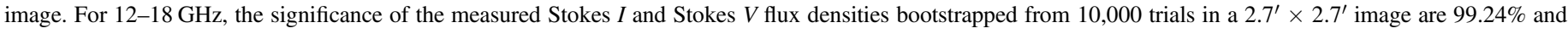

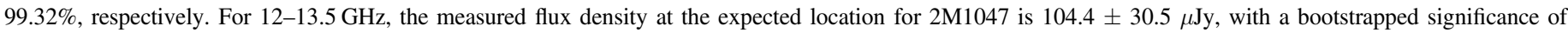

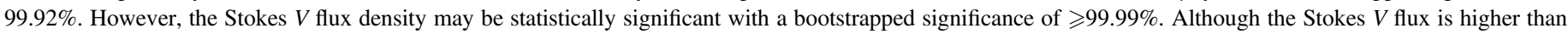
the measured flux for Stokes $I$, the discrepancy is within the rms noise. We classify these detections as tentative.

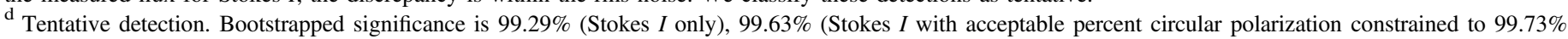

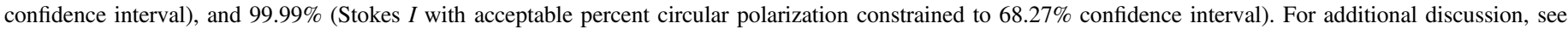
Secition 4.2 .

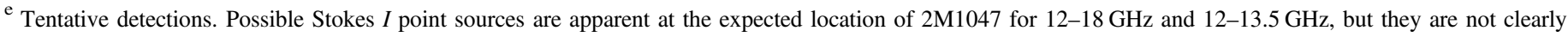
distinguishable by eye from the rms noise image. Bootstrapped significance levels are $99.59 \%$ and $99.98 \%$, respectively.

polarized $(\sim 35 \%)$ quiescent emission $(27.8 \pm 1.3 \mu \mathrm{Jy}$ mean flux density) present throughout the entire $8-12 \mathrm{GHz}$ band, as well as Pulse 2, a very bright $(159.7 \pm 5.3 \mu \mathrm{Jy}$ mean flux density) and highly circularly polarized ( $\sim 80 \%)$ pulse occurring near the center of the observation time window. Pulse 2 is observable at all sub-bands within the full $8-12 \mathrm{GHz}$ band, although its flux density varies from band to band by a factor of nearly 3 (see Section 6.2 for discussion about such frequency-dependent variability). Two substantially weaker pulses with mean flux densities $41.3 \pm 5.4 \mu \mathrm{Jy}$ and $61.0 \pm 5.7 \mu \mathrm{Jy}$ additionally occur before and after Pulse 2. Figure 3 shows the time series for $2 \mathrm{M} 1237$, and we report the characteristics of the pulsed and quiescent emission in Table 5.

Such strong pulses suggested a straightforward period analysis, and indeed, the periods returned by the L-S, Plavchan, and BLS periodogram algorithms are consistent within the uncertainties (see Table 9). However, the data for 2M1237 do not appear to provide enough phase coverage to adequately sample periods longer than $\sim 3.77 \mathrm{hr}$. Plavchan peak power locations at and longer than this $\sim 3.77 \mathrm{hr}$ period change dramatically depending on input variables and especially on the fractional amount of outliers (Figure 5). Specifically, Plavchan periodograms with a lower fraction of allowed outliers are biased in favor of a period that is approximately twice longer than the periods favored when allowed outlier fractions are higher. This occurs because the flux density of Pulse 2 deviates strongly from the mean amplitude of the smaller pulses before and after it. When the algorithm is not allowed to ignore data points from this strong pulse, it will favor a rotation period that generates a time series similar to one with a main transit and a 
Table 7

SIMP0136 and 2M1043: Pulsed and Quiescent Emission

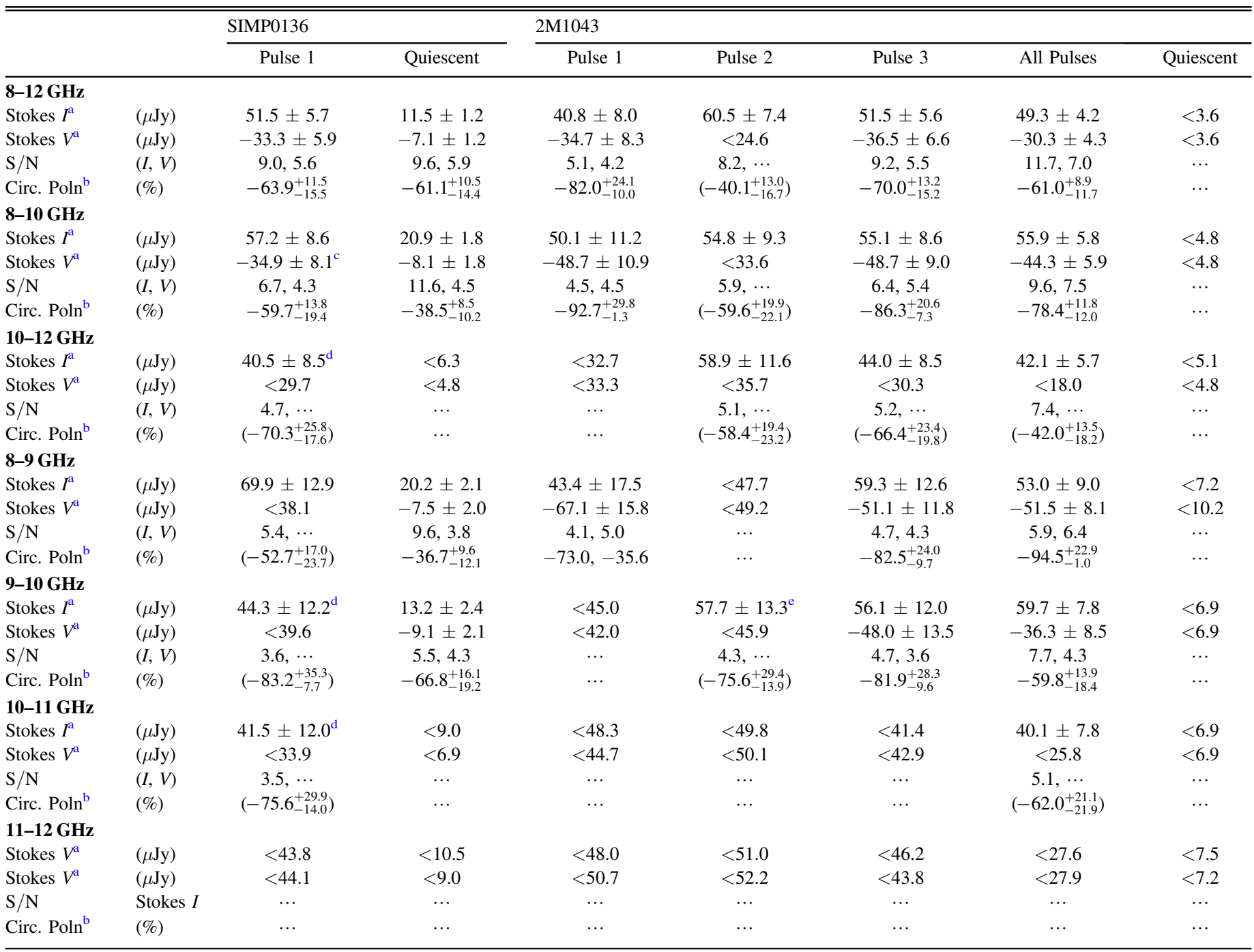

Notes.

${ }^{a}$ Reported flux densities are integrated over the FWHM of the full-bandwidth $60 \mathrm{~s}$ resolution data. Fixing fit parameters can result in overestimated uncertainties on the integrated and peak flux densities, so we report the rms image noise as the uncertainty $\sigma_{\text {rms }}$. For targets with a clear visual non-detection, we list $3 \sigma_{\text {rms }}$.

${ }^{\mathrm{b}}$ Reported polarization fractions are highest-likelihood values, given the measured Stokes $I$ and Stokes $V$ flux densities. Uncertainties reflect upper and lower bounds of $68.27 \%$ confidence intervals. Negative and positive values indicate left- and right- circular polarizations, respectively. Upper-bound $68.27 \%$ and $99.73 \%$ confidence intervals are given for sub-bands with $-100 \%$ circular polarization. Lower bounds are given in parentheses for objects without detectable levels of Stokes $V$ emission, assuming a $3 \sigma_{\text {rms }}$ flux density and left circular polarization.

${ }^{\mathrm{c}}$ Tentative image detection (no clearly visually distinguishable Stokes $V$ point source). Bootstrapped significance is $99.67 \%$.

d Tentative image detection (no clearly visually distinguishable Stokes I point source). Bootstrapped significance is $99.66 \%$ (10-12 GHz), 98.78\% (9-10 GHZ), $98.80 \%$ (10-11 GHZ).

${ }^{\mathrm{e}}$ Tentative image detection (no clearly visually distinguishable Stokes $I$ point source). Bootstrapped significance is $99.54 \%$.

secondary eclipse. Additional phase coverage to characterize the variable behavior of the pulse profile is necessary to resolve the ambiguity between period harmonics.

2 MASS $10475385+2124234$. We detect 2 M1047 in initial Stokes $I$ imaging with an $\mathrm{S} / \mathrm{N}$ of 16.8 . In contrast, there is no clear Stokes $V$ detection, with an $\mathrm{S} / \mathrm{N}$ of only 2.6. Table 4 gives the measured mean flux density and rms noise. Highly circularly polarized pulses are clearly evident in the 10,60 , and 120 s subband time series for 2M1047, with two large-amplitude pulses occurring near the beginning of the observation time window (Pulse 1 and Pulse 2). Pulse 1 occurred during a time range when strong RFI caused all $12-12.8 \mathrm{GHz}$ data to be flagged, affecting noise properties and especially so for the $12-13.5 \mathrm{GHz}$ sub-band. To check if Pulse 1 could be attributed to this additional noise, we created time series for a nearby object at $10^{\mathrm{h}} 47^{\mathrm{m}} 54^{\mathrm{s}} .95+$ $21^{\circ} 24^{\prime} 13$." $40 \mathrm{~s}$ and searched for variability that correlates with Pulse 1. We include this comparison time series in the $2 \mathrm{M} 1047$ time-series figures for $120 \mathrm{~s}$ resolution. This comparison object 
Table 8

SDSS0423: Pulsed and Quiescent Emission

\begin{tabular}{|c|c|c|c|c|c|c|c|c|}
\hline & & Pulse R1 & Pulse R2 & Pulse L1 & Pulse L2 & Pulse L3 & Pulse L4 & Quiescent \\
\hline \multicolumn{9}{|l|}{ 8-12 GHz } \\
\hline Stokes $I^{\mathrm{a}}$ & $(\mu \mathrm{Jy})$ & $86.9 \pm 9.6$ & $82.0 \pm 9.5$ & $99.2 \pm 8.2$ & $58.0 \pm 6.6$ & $64.6 \pm 5.0$ & $101.0 \pm 9.1$ & $<5.1$ \\
\hline Stokes $V^{\mathrm{a}}$ & $(\mu \mathrm{Jy})$ & $<29.7$ & $<24.0$ & $-94.2 \pm 6.7$ & $-37.0 \pm 7.0$ & $-34.3 \pm 4.6$ & $-99.3 \pm 10.1$ & $<5.7$ \\
\hline $\mathrm{S} / \mathrm{N}$ & $(I, V)$ & $9.1, \cdots$ & $8.6, \cdots$ & $12.1,14.1$ & $10.1,7.6$ & $12.9,7.5$ & $11.1,9.8$ & $\ldots$ \\
\hline Circ. Poln ${ }^{\mathrm{b}}$ & $(\%)$ & $\left(33.8_{-11.0}^{+13.5}\right)$ & $\left(28.9_{-9.4}^{+11.8}\right)$ & $-94.3_{-2.8}^{+10.9}$ & $-63.0_{-16.0}^{+12.1}$ & $-52.8_{-9.3}^{+7.4}$ & $-81.8,-62.2$ & $\cdots$ \\
\hline \multicolumn{9}{|l|}{ 8-10 GHz } \\
\hline Stokes $I^{\mathrm{a}}$ & $(\mu \mathrm{Jy})$ & $90.2 \pm 11.4$ & $96.5 \pm 10.6$ & $121.4 \pm 11.7$ & $69.3 \pm 8.7$ & $82.6 \pm 6.0$ & $152.6 \pm 13.3$ & $<6.6$ \\
\hline Stokes $V^{\mathrm{a}}$ & $(\mu \mathrm{Jy})$ & $51.9 \pm 10.9$ & $<34.5$ & $-132.3 \pm 12.5$ & $-67.1 \pm 9.8$ & $-49.6 \pm 6.0$ & $-151.9 \pm 15.8$ & $<6.6$ \\
\hline $\mathrm{S} / \mathrm{N}$ & $(I, V)$ & $7.9,4.8$ & $9.1, \cdots$ & $10.4,10.6$ & $8.0,6.8$ & $13.8,8.3$ & $11.5,9.6$ & $\ldots$ \\
\hline Circ. Poln ${ }^{\mathrm{b}}$ & $(\%)$ & $56.6_{-11.8}^{+16.8}$ & $\left(35.3_{-11.5}^{+14.1}\right)$ & $-86.4,-68.0$ & $-95.3_{-0.7}^{+20.6}$ & $-59.7_{-9.6}^{+7.6}$ & $-82.3,-62.5$ & $\cdots$ \\
\hline \multicolumn{9}{|l|}{ 10-12 GHz } \\
\hline Stokes $I^{\mathrm{a}}$ & $(\mu \mathrm{Jy})$ & $83.7 \pm 14.5$ & $56.5 \pm 13.8$ & $67.2 \pm 12.9^{\mathrm{d}}$ & $<10.5$ & $53.1 \pm 8.8$ & $<41.7$ & $<7.2$ \\
\hline Stokes $V^{\mathrm{a}}$ & $(\mu \mathrm{Jy})$ & $<39.9$ & $<39.0$ & $<37.5$ & $<30.3$ & $<23.4$ & $<45.9$ & $<7.5$ \\
\hline $\mathrm{S} / \mathrm{N}$ & $(I, V)$ & $5.8, \cdots$ & $4.1, \cdots$ & $5.2, \cdots$ & $\ldots$ & $6.0, \cdots$ & $\ldots$ & $\ldots$ \\
\hline Circ. Poln ${ }^{\mathrm{b}}$ & $(\%)$ & $\left(46.3_{-14.7}^{+22.2}\right)$ & $\left(65.2_{-23.0}^{+21.0}\right)$ & $\left(-53.8_{-24.0}^{+17.4}\right)$ & $\ldots$ & $\left(-42.9_{-55.4}^{+42.9}\right)$ & $\cdots$ & $\cdots$ \\
\hline \multicolumn{9}{|l|}{ 8-9 GHz } \\
\hline Stokes $I^{\mathrm{a}}$ & $(\mu \mathrm{Jy})$ & $73.8 \pm 18.5$ & $111.6 \pm 14.0$ & $133.5 \pm 16.3$ & $72.2 \pm 12.9$ & $95.5 \pm 8.9$ & $218.1 \pm 21.0$ & $<8.4$ \\
\hline Stokes $V^{\mathrm{a}}$ & $(\mu \mathrm{Jy})$ & $65.7 \pm 16.0^{\mathrm{c}}$ & $<44.1$ & $-166.7 \pm 16.1$ & $-78.5 \pm 13.7$ & $-52.8 \pm 9.1$ & $-209.9 \pm 21.0$ & $<8.4$ \\
\hline $\mathrm{S} / \mathrm{N}$ & $(I, V)$ & $4.0,4.1$ & $8.0, \cdots$ & $8.2,10.4$ & $5.6,5.7$ & $10.7,5.8$ & $10.4,10.0$ & $\ldots$ \\
\hline Circ. Poln ${ }^{\mathrm{b}}$ & $(\%)$ & $83.9_{-26.6}^{+8.3}$ & $\left(38.9_{-12.6}^{+16.4}\right)$ & $-88.4,-70.2$ & $-73.4,-42.1$ & $-54.8_{-12.5}^{+9.5}$ & $-95.4_{-1.5}^{+14.8}$ & $\ldots$ \\
\hline \multicolumn{9}{|l|}{ 9-10 GHz } \\
\hline Stokes $I^{\mathrm{a}}$ & $(\mu \mathrm{Jy})$ & $110.2 \pm 19.0$ & $93.6 \pm 14.8$ & $102.3 \pm 15.9$ & $60.6 \pm 12.0$ & $69.6 \pm 8.8$ & $86.5 \pm 18.2$ & $<8.7$ \\
\hline Stokes $V^{\mathrm{a}}$ & $(\mu \mathrm{Jy})$ & $<54.6$ & $<46.8$ & $-103.3 \pm 15.3$ & $-56.5 \pm 12.4$ & $-49.8 \pm 8.5$ & $-107.0 \pm 21.0$ & $<8.7$ \\
\hline $\mathrm{S} / \mathrm{N}$ & $(I, V)$ & $5.8, \cdots$ & $6.3, \cdots$ & $6.4,6.8$ & $5.0,4.6$ & $7.9,5.9$ & $4.8,5.1$ & $\ldots$ \\
\hline Circ. Poln ${ }^{\mathrm{b}}$ & $(\%)$ & $\left(48.1_{-15.3}^{+22.6}\right)$ & $\left(48.8_{-15.7}^{+21.8}\right)$ & $-74.7,-47.8$ & $-89.8_{-10.2}^{+62.8}$ & $-70.4_{-15.6}^{+12.8}$ & $-72.9,-36.8$ & $\ldots$ \\
\hline \multicolumn{9}{|l|}{ 10-11 GHz } \\
\hline Stokes $I^{\mathrm{a}}$ & $(\mu \mathrm{Jy})$ & $82.7 \pm 17.9$ & $<52.8$ & $<49.5$ & $<39.0$ & $<31.5$ & $<65.7$ & $<8.4$ \\
\hline Stokes $V^{\mathrm{a}}$ & $(\mu \mathrm{Jy})$ & $<57.0$ & $<48.9$ & $<48.0$ & $<38.7$ & $<30.3$ & $<60.3$ & $<8.4$ \\
\hline $\mathrm{S} / \mathrm{N}$ & $(I, V)$ & $4.6, \cdots$ & $\cdots$ & $\ldots$ & $\ldots$ & $\ldots$ & $\ldots$ & $\cdots$ \\
\hline Circ. Poln ${ }^{\mathrm{b}}$ & $(\%)$ & $\left(65.9_{-23.3}^{+20.3}\right)$ & $\ldots$ & $\ldots$ & $\ldots$ & $\ldots$ & $\ldots$ & $\ldots$ \\
\hline \multicolumn{9}{|l|}{ 11-12 GHz } \\
\hline Stokes $I^{\mathrm{a}}$ & $(\mu \mathrm{Jy})$ & $<71.4$ & $<66.0$ & $<88.2$ & $<51.3$ & $<39.9$ & $<75.3$ & $<12.9$ \\
\hline Stokes $V^{\mathrm{a}}$ & $(\mu \mathrm{Jy})$ & $<72.6$ & $<65.7$ & $<97.2$ & $<51.3$ & $<38.1$ & $<76.5$ & $<16.2$ \\
\hline $\mathrm{S} / \mathrm{N}$ & $(I, V)$ & $\ldots$ & $\ldots$ & $\ldots$ & $\ldots$ & $\ldots$ & $\ldots$ & $\ldots$ \\
\hline Circ. Poln ${ }^{\mathrm{b}}$ & $(\%)$ & $\ldots$ & $\ldots$ & $\ldots$ & $\ldots$ & $\ldots$ & $\ldots$ & $\ldots$ \\
\hline
\end{tabular}

Notes.

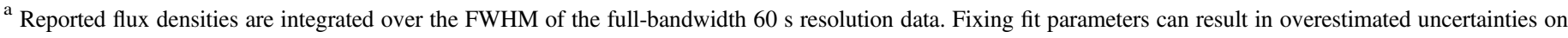
the integrated and peak flux densities, so we report the rms image noise as the uncertainty $\sigma_{\text {rms. }}$. For targets with a clear visual non-detection, we list $3 \sigma_{\text {rms }}$.

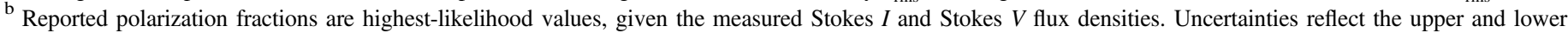

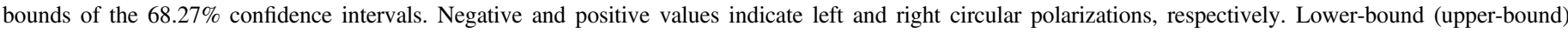

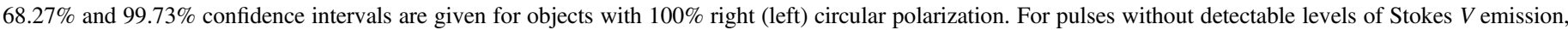

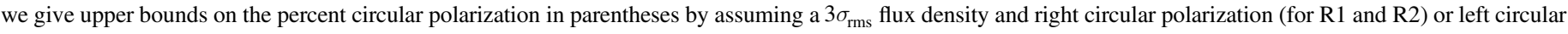
polarization (for L1-L4).

c Tentative image detection (no clearly visually distinguishable Stokes $V$ point source). Bootstrapped significance is $99.27 \%$.

d Tentative Stokes $I$ image detection is difficult to distinguish from image noise. Bootstrapped significance is $\geqslant 99.99 \%$.

does not exhibit any evidence of highly circularly polarized pulses at any of the frequencies or time stamps associated with the pulses detected for $2 \mathrm{M} 1047$.

When checking each pulse individually with imaging, Pulses 3-5 were very faint and were difficult to individually distinguish by eye in the imaging (see Figure 1). To further check these pulses, we averaged them together to reduce rms noise and report measured flux densities for this averaged image in Table 6. Pulses 3-5 were clearly detectable by eye in the $12-18 \mathrm{GHz}$ and $15-16.5 \mathrm{GHz}$ images.

Pulse 5 may extend into the $16.5-18 \mathrm{GHz}$ time series. We measured Stokes $I$ and Stokes $V$ flux densities of $91.5 \pm$
$28.7 \mu \mathrm{Jy}$ and $-94.9 \pm 24.9 \mu \mathrm{Jy}$, respectively, where negative values indicate left-circular polarization. The percent circular polarization is expected to lie between $[-100 \%,-58.0 \%]$ with $68.27 \%$ confidence and $[-100 \%,-14.3 \%]$ with $99.73 \%$ confidence. However, there is no clear point source in the associated images. The bootstrapped Stokes $I$ significance is $99.29 \%$. The significance increases to $99.63 \%$ and $99.99 \%$ when we constrain the acceptable percent circular polarization to lie within the $99.73 \%$ and $68.27 \%$ confidence intervals, respectively. We classify the $16.5-18 \mathrm{GHz}$ detection as a tentative detection. We report the characteristics of the pulsed and quiescent emission in Table 6. 
Table 9

Periodogram Results

\begin{tabular}{|c|c|c|c|c|}
\hline Object & $\begin{array}{l}\mathrm{L}-\mathrm{S} \\
(\mathrm{hr})\end{array}$ & $\begin{array}{c}\text { Plavchan } \\
\text { (hr) }\end{array}$ & $\begin{array}{c}\text { BLS } \\
(\mathrm{hr})\end{array}$ & $\begin{array}{c}\text { Adopted } \\
\text { (hr) }\end{array}$ \\
\hline $2 \mathrm{M} 1047^{\mathrm{a}}$ & $0.59_{-0.02}^{+0.02}$ & $1.78_{-0.06}^{+0.07}$ & $1.77_{-0.05}^{+0.05}$ & $1.78_{-0.06}^{+0.07}$ \\
\hline SIMP0136 ${ }^{\mathrm{b}}$ & $2.33_{-0.32}^{+0.43}$ & $2.88_{-0.27}^{+0.34}$ & $2.74_{-0.50}^{+0.80}$ & $2.88_{-0.27}^{+0.34}$ \\
\hline 2M1043 & $2.36_{-0.31}^{+0.42}$ & $2.19_{-0.12}^{+0.15}$ & $2.21_{-0.13}^{+0.14}$ & $2.21_{-0.13}^{+0.14}$ \\
\hline $2 \mathrm{M} 1237$ & $2.21_{-0.39}^{+0.59}$ & $2.28_{-0.09}^{+0.10}$ & $2.28_{-0.12}^{+0.13}$ & $2.28_{-0.09}^{+0.10}$ \\
\hline SDSS0423 & $1.44_{-0.15}^{+0.19}$ & $1.49_{-0.10}^{+0.11}$ & $1.47_{-0.11}^{+0.13}$ & $1.47_{-0.11}^{+0.13}$ \\
\hline
\end{tabular}

Notes.

${ }^{a}$ No periodicity is clearly observable in the pulsed emission from 2M1047. The detected periodicity is consistent with the $\sim 1.77 \mathrm{hr}$ C-band pulse period measured by Williams \& Berger (2015), suggesting that our detected periodicity may be due to the pulsed emission and/or the quiescent emission. For our discussion in Section 6.3, we adopt the rotation period measured by Williams \& Berger (2015).

${ }^{b}$ No periodicity is observable in the pulsed emission from SIMP0136. The periods listed here correspond to the non-pulsed quasi-quiescent emission. For our discussion in Section 6.3, we adopt the photometric rotation period $P=2.3895 \pm 0.0005 \mathrm{hr}$ measured by Croll et al. (2016).

When applying the periodogram analyses, 2M1047 stood out as the sole object whose periods returned by the $\mathrm{L}-\mathrm{S}$, Plavchan, and BLS algorithms were inconsistent with each other (see Table 9 and Figure 5). The L-S periodogram returns a $\sim 0.59 \mathrm{hr}$ period, while Plavchan returns $\sim 1.77 \mathrm{hr}$, and BLS returns either $\sim 3.54 \mathrm{hr}$ or $\sim 1.77 \mathrm{hr}$ depending on the maximum allowed rotation pulse phase coverage and phase binning. Fortunately, these periods are all harmonics, suggesting a non-spurious origin. Similar to 2M1237, the longest period is favored by the BLS algorithm for the cases with the least number of data points per bin, emphasizing the significance of the strongest peaks. The Plavchan periodogram also reflects this behavior, although its most significant period is consistently $\sim 1.77 \mathrm{hr}$, regardless of input parameters. For ground-based transit surveys, a typical number of points per bin is of the order of a few tens to a hundred, which would correspond to a $\sim 1.77 \mathrm{hr}$ period.

Owing to the observed intermittency of the pulses, the periodogram results are tantalizing, but inconclusive. However, the periodogram detects periodicity consistent with the expected period as measured by Williams \& Berger (2015) using $10 \mathrm{hr} \mathrm{C}$-band (4-6 GHz) observations, suggesting that our detected periodicity may be due to the pulsed emission and/or the quiescent emission. Given the ambiguities arising from the periodogram analysis of 2M1047 and the lack of clear pulse periodicity in the phase-folded light curves, we treat the periodogram analysis as a confirmation of the period measured by Williams \& Berger (2015).

SIMP J01365662+0933473. We detect SIMP0136 in initial Stokes $I$ and Stokes $V$ imaging with an S/N of 65.9 and 21.6, respectively. Table 4 gives the measured mean flux density and rms noise. SIMP0136 appears to have broadly variable quasiquiescent radio emission with a single broad peak (Pulse 1) that is persistent across 60 and $120 \mathrm{~s}$ sub-band time series (see Figure 2). We confirm Pulse 1 with imaging and report the characteristics of the pulsed and quiescent emission in Table 7.

At first glance, the 8-12 GHz time-averaged quasi-quiescent emission from SIMP0136 is similarly circularly polarized as for Pulse $1(\sim 60 \%)$. Upon closer examination, Pulse 1 is more strongly circularly polarized than the quasi-quiescent emission at $8-10 \mathrm{GHz}(\sim 60 \%$ versus $\sim 40 \%)$. At the $10-12 \mathrm{GHz}$ subband, any Stokes $V$ emission that may be present cannot be distinguished from the rms noise for either Pulse 1 or the quasiquiescent emission. Although the $10-12 \mathrm{GHz}$ Pulse 1 detection is tentative $(40.5 \pm 8.5 \mu \mathrm{Jy}$ with $99.67 \%$ bootstrapped significance), it is important to note that the quasi-quiescent emission is undetectable in Stokes $I$ down to a $3 \sigma_{\text {rms }}$ level of $6.2 \mu \mathrm{Jy}$. The significantly lower rms noise results from the longer time coverage of the quasi-quiescent emission as compared to the narrower time-width of Pulse 1 . When we further examine the SIMP0136 time series at $1 \mathrm{GHz}$ bandwidths, the Stokes $I$ detection remains clear for Pulse 1 at $8-9 \mathrm{GHz}(69.9 \pm 12.9 \mu \mathrm{Jy})$ and becomes more tentative at $9-10 \mathrm{GHz}$ and $10-11 \mathrm{GHz} \quad(44.3 \pm 12.2 \mu \mathrm{Jy}$ with $98.78 \%$ bootstrapped significance and $41.5 \pm 12.0 \mu \mathrm{Jy}$ with $98.80 \%$ bootstrapped significance, respectively), finally becoming indistinguishable from rms noise at $11-12 \mathrm{GHz}$. These tentative detections are further bolstered by measured flux densities that are consistent with those measured for the $8-10 \mathrm{GHz}$ and 10-12 GHz sub-bands. In contrast to the persistence of Pulse 1 emission up through $11 \mathrm{GHz}$, the Stokes $I$ quasi-quiescent emission becomes undetectable above $10 \mathrm{GHz}$, at $3 \sigma_{\text {rms }}$ noise levels of 9.0 and $10.5 \mu \mathrm{Jy}$. Given these comparisons, we are confident of the $8-9 \mathrm{GHz}$ Pulse 1 detection and classify the 9-10 GHz and $10-11 \mathrm{GHz}$ detections as tentative.

Infrared cloud variability studies of SIMP0136 suggest that its rotation period is $P=2.3895 \pm 0.0005 \mathrm{hr}$. This a priori knowledge of the expected pulse periodicity allows us to search for pulses at expected occurrence times in our observing block. A pulse occurring before the above-noted time series peak would have directly coincided with a phase calibrator observation and thus possibly prevented its detection. A pulse occurring after would have taken place near the middle of the target integration block, when phase errors would be greatest and might smear out flux from a pulse. To check for the effects of phase errors on flux densities, we imaged a bright nearby object at $01^{\mathrm{h}} 36^{\mathrm{m}} 47^{\mathrm{s}} .63 \mathrm{~s}+09^{\circ} 34^{\prime} 04^{\prime \prime} .25$ and well within the 4.5 primary beam during "edge" and "middle" observing scans. "Edge" scans are directly adjacent to a phase calibration scan, whereas "middle" scans are sandwiched by the edge scans and therefore likely suffer from the worst phase calibration errors. We measured only a $3.2 \pm 1.8 \%$ decrease in flux, suggesting that phase calibration errors cannot account for a possible missing pulse. We conclude that either another pulse exists but is not detectable, or there is no other pulse. See Section 6.2 for an in-depth discussion.

Despite the single pulse, we include SIMP0136 in the periodogram analysis for the sake of completeness. The period returned by the $\mathrm{L}-\mathrm{S}$, Plavchan, and BLS algorithms are consistent with each other within the uncertainties, and appear to be based on the variability occurring in the quasi-quiescent emission. We adopt a period of $2.88_{-0.27}^{+0.34} \mathrm{hr}$ for the quasiquiescent emission at $\mathrm{X}$ band. We analyzed the $4-8 \mathrm{GHz}$ data from Kao et al. (2016) and find that the C-band period appears nominally consistent with $\sim 2.88 \mathrm{hr}$, but the data are inconclusive because the total C-band observing block was only $4 \mathrm{hr}$ long. In contrast to the X-band period, the photometric period is $2.3895 \pm 0.0005 \mathrm{hr}$. These periods are not statistically distinct.

With only one visually apparent ECM pulse, we cannot confirm a cloud-independent rotation period for SIMP0136. Since ECM emission is more clearly discerned at $4-8 \mathrm{GHz}$ for 
SIMP0136, we recommend a future rotation study using longduration observations at $4-8 \mathrm{GHz}$ to determine the cloudindependent rotation period of SIMP0136. Because the mechanism generating the non-pulsed but varying quiescent emission and its location within the brown dwarf system remain unknown, while the infrared variability is expected to occur within the brown dwarf atmosphere, we adopt the rotation period measured by photometric studies for our discussion in Section 6.

2MASS J10430758+2225236. We detect $2 \mathrm{M} 1043$ in initial Stokes $I$ imaging with an $\mathrm{S} / \mathrm{N}$ of 9.5 . The Stokes $V$ detection is very faint, with an $\mathrm{S} / \mathrm{N}$ of 4.7 . Table 4 gives the measured mean flux density and rms noise. In its time series, 2M1043 has three very faint pulses that become clearly evident when the data are averaged across the full $8-12 \mathrm{GHz}$ bandwidth (see Figure 2). At the full $4 \mathrm{GHz}$ bandwidth, the pulses have flux densities that range from $40.8 \pm 8.0 \mu \mathrm{Jy}$ through $60.5 \pm$ $7.4 \mu \mathrm{Jy}$. When imaged individually, these pulses are difficult to distinguish by eye in the imaging. To reduce the rms noise, we averaged the three pulses together to check for them in subband imaging. We include measured flux densities for these averaged images in the "All Pulses" column in Table 6.

In the time series, the pulses are most clearly visually evident at the $8-12 \mathrm{GHz}$ and $8-10 \mathrm{GHz}$ bands. In the imaging, the pulses remain evident through the $9-10 \mathrm{GHz}$ sub-band for both Stokes $I$ and Stokes $V$. At $10-11 \mathrm{GHz}$, the Stokes $I$ component of the averaged-together pulses remains clear with flux density $40.1 \pm 7.8 \mu \mathrm{Jy}$, but the Stokes $V$ component is undetectable up to a $3 \sigma_{\text {rms }}$ flux density of $25.8 \mu \mathrm{Jy}$ when all three pulses are averaged together. When judging if these pulses are truly present or not, we compared the "All Pulses" flux density measurements in each $1 \mathrm{GHz}$ sub-band to the flux density measurements for quiescent emission. In contrast to clear Stokes I pulsed emission up through 10-11 GHz, 2M1043 does not appear to have any detectable quiescent emission $\geqslant 6.9 \mu \mathrm{Jy}$ $\left(3 \sigma_{\mathrm{rms}}\right)$ in that sub-band, or $\geqslant 3.6 \mu \mathrm{Jy}\left(3 \sigma_{\mathrm{rms}}\right)$ for the full 8-12 GHz bandwidth. We therefore conclude that the pulses are present through the $10-11 \mathrm{GHz}$ sub-band.

The periods returned by the $\mathrm{L}-\mathrm{S}$, Plavchan, and BLS periodogram algorithms are consistent within the uncertainties. Given the sharpness of the pulses, we rule out the period returned by the $\mathrm{L}-\mathrm{S}$ algorithm as our adopted period. This is because the $\mathrm{L}-\mathrm{S}$ algorithm relies on Fourier analysis and therefore is not well suited to time series with sharp pulses, which require many high-order sinusoids to reproduce. Following the method we outlined in Section 4.3, we adopt the period returned by BLS, which results in a folded time series with the most visual agreement in pulse overlaps.

SDSS J04234858-0414035. We detect SDSS0423 in initial Stokes $I$ imaging with an $\mathrm{S} / \mathrm{N}$ of 12.9 and no Stokes $V$ detection. Table 4 gives the measured mean flux density and rms noise. SDSS0423 has four left-circularly polarized pulses that are clearly evident through $10 \mathrm{GHz}$. At $8-9 \mathrm{GHz}$ and $9-10 \mathrm{GHz}$, the peak flux density ranges from $60.6 \pm 12.0 \mu \mathrm{Jy}$ for the faintest pulse to $218.1 \pm 21.0 \mu \mathrm{Jy}$ for the brightest pulse. At these frequency ranges, the pulses are strongly circularly polarized, with highest-likelihood percent polarizations between $-54.8 \%$ and $-95.4 \%$. At $10-11 \mathrm{GHz}$ and $11-12 \mathrm{GHz}$, these pulses fade and become undetectable up to Stokes $I 3 \sigma_{\text {rms }}$ limits between $31.5 \mu \mathrm{Jy}$ and $88.2 \mu \mathrm{Jy}$. However, when the left-circularly polarized pulses are averaged over $2 \mathrm{GHz}$ bandwidths, Pulses
L1 and L3 remain clearly detectable in Stokes $I$ with flux densities of $67.2 \pm 12.9 \mu \mathrm{Jy}$ and $53.1 \pm 8.8 \mu \mathrm{Jy}$, respectively.

In addition to the left-circularly polarized pulses, there are two fainter right-circularly polarized pulses, with peak Stokes $I$ flux densities between $73.8 \pm 18.5 \mu \mathrm{Jy}$ and $111.6 \pm 14.0 \mu \mathrm{Jy}$ throughout the 8-9 GHz and 9-10 GHz bands. Except for Pulse $\mathrm{R} 1$ at $8-9 \mathrm{GHz}$, these right-circularly polarized pulses are less strongly polarized than the left-circularly polarized pulses. They are undetectable in Stokes $V$ up to $3 \sigma_{\text {rms }}$ limits between $44.1 \mu \mathrm{Jy}$ and $54.6 \mu \mathrm{Jy}$, with corresponding upper limits on the highest-likelihood percent circular polarization between $38.9 \%$ and $48.8 \%$. Pulse R1 at $8-9 \mathrm{GHz}$ is strongly polarized, with a Stokes $V$ flux density of $65.7 \pm 16.0 \mu \mathrm{Jy}$ and a highestlikelihood percent circular polarization of $83.9 \%$. At 10-11 GHz, only Pulse R1 remains detectable in Stokes $I$, with a flux density of $82.7 \pm 17.9 \mu \mathrm{Jy}$. However, its Stokes $V$ flux density fades and cannot be detected above a $3 \sigma_{\text {rms }}$ limit of $57.0 \mu \mathrm{Jy}$. At $11-12 \mathrm{GHz}$, both right-circularly polarized pulses become undetectable above a Stokes I $3 \sigma$ limit between $66.0 \mu \mathrm{Jy}$ and $71.4 \mu \mathrm{Jy}$.

With stronger left-circularly polarized pulses than rightcircularly polarized pulses, these X-band observations directly contrast with the C-band observations for SDSS0423, in which the right-circularly polarized pulses are stronger than the leftcircularly polarized ones (Kao et al. 2016). Also in contrast to its C-band behavior, SDSS0423 does not appear to have any detectable quiescent emission above a Stokes $I 3 \sigma_{\mathrm{rms}}$ limit of $\sim 5.1 \mu \mathrm{Jy}$ for the full $4 \mathrm{GHz}$ bandwidth (see Section 6.1 for a discussion).

The multiple pulses in the SDSS0423 time series allow for a straightforward periodogram analysis. The periods returned by the L-S, Plavchan, and BLS periodogram algorithms are consistent within the uncertainties (see Table 9). Following the method we outlined in Section 4.3 , we adopt the $\sim 1.47 \mathrm{hr}$ period returned by BLS, which results in a folded time series with the most visual agreement in pulse overlaps. This period is consistent with the $2.0 \pm 0.4 \mathrm{hr}$ J-band variability period reported by Clarke et al. (2008). Additionally, with a $v \sin i=60 \pm 10 \mathrm{~km} \mathrm{~s}^{-1}$ (Prato et al. 2015), the corresponding lower-bound radius is $R \sin i=0.71 \pm 0.13 R_{\mathrm{J}}$. This lowerbound radius is consistent with the $\sim 0.9-1.0 R_{\mathrm{J}}$ radii inferred from dynamical masses measured by Dupuy \& Liu (2017).

\section{Discussion}

\subsection{The Curious Case of Highly Circularly Polarized and/or Disappearing Quiescent Emission}

Kao et al. (2016) noted that all known radio brown dwarfs exhibited detectable levels of quiescent emission, and Pineda et al. (2017) showed that the quiescent radio luminosities correlated with $\mathrm{H} \alpha$ luminosities for confirmed auroral emitters (i.e. with clear rotational modulation in the highly circularly polarized radio emission component). This suggested a possible connection between pulsed and quiescent radio processes.

In contrast, we do not observe detectable levels of quiescent emission from SDSS0423 and $2 \mathrm{M} 1043$ for $8-12 \mathrm{GHz}$ or individual 1 or $2 \mathrm{GHz}$ sub-bands, down to $3 \sigma_{\text {rms }}$ noise levels of $\sim 5.1-12.9 \mu \mathrm{Jy}$ and $\sim 3.6-7.5 \mu \mathrm{Jy}$, respectively. We also do not observe detectable quiescent emission from 2M1047 at frequencies $\gtrsim 13.5 \mathrm{GHz}$ down to $3 \sigma_{\text {rms }}$ noise levels of $\sim 3.5-5.2 \mu \mathrm{Jy}$. For SDSS0423, Kao et al. (2016) measured a 

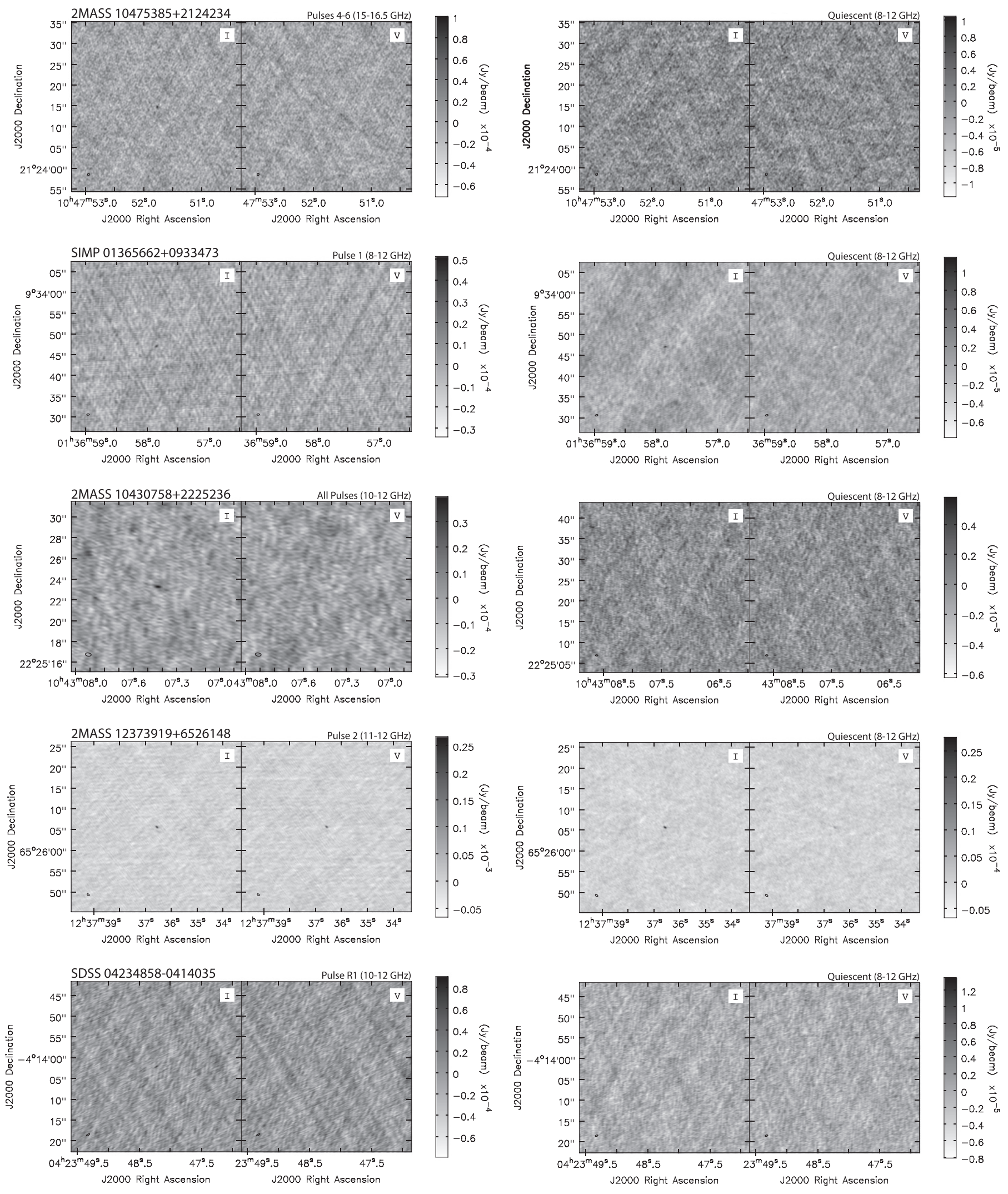

Figure 4. Stokes $I$ and Stokes $V$ images of pulsed emission (left) and quiescent emission (right). Images are centered over measured target coordinates, and ellipses in bottom left corners depict synthesized beam dimensions. No quiescent emission is detectable from 2M1043 or SDSS0423. A measurement of the flux density at the expected coordinates for $2 \mathrm{M} 1047$ yields a tentative detection, but a point source is not clearly distinguishable by eye. 

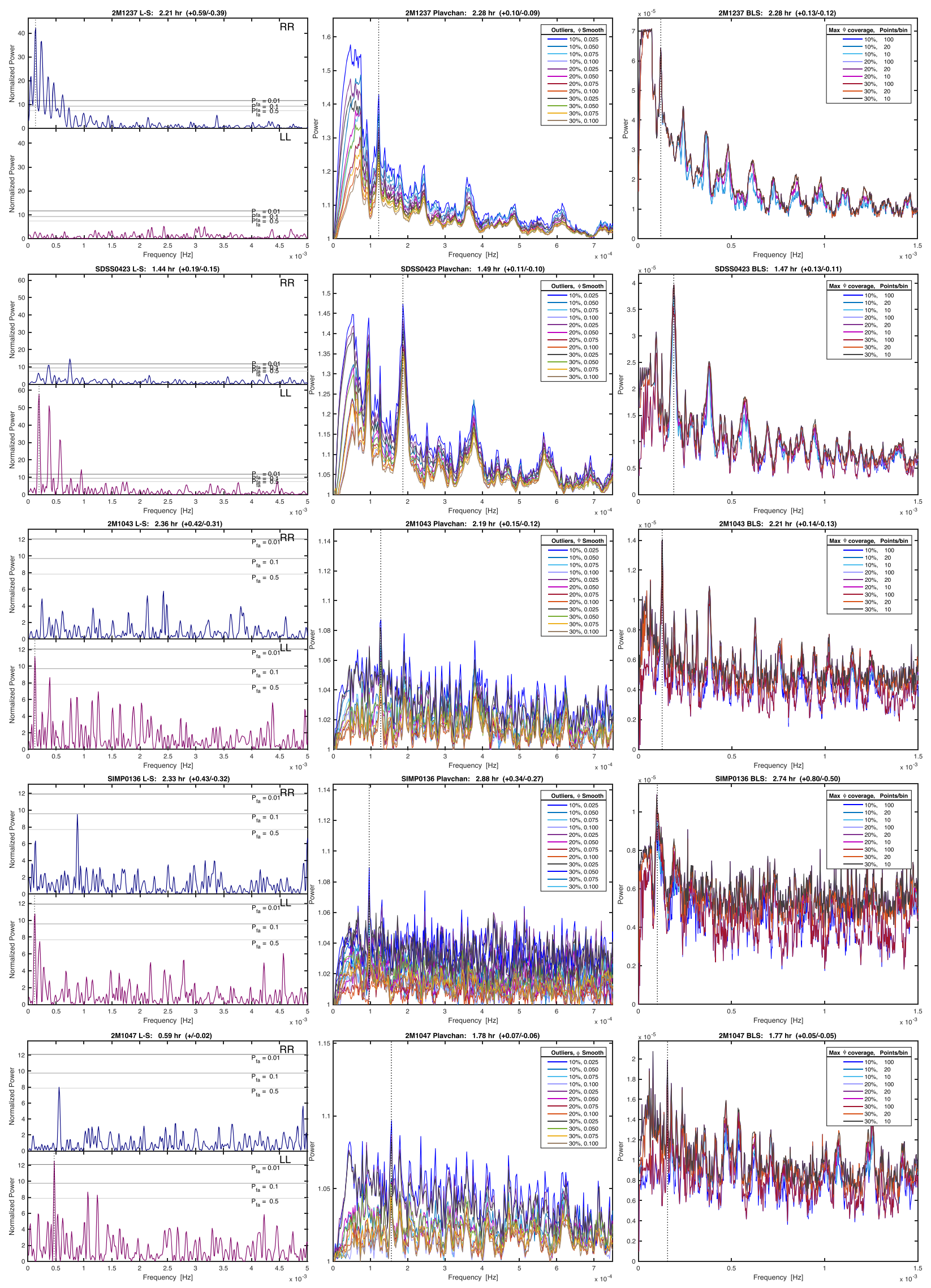

Figure 5. From left to right: L-S, Plavchan, and BLS periodograms. RR and LL periodograms are shown for the L-S periodogram to show relative powers of peaks between time series with and without apparent periodic variation. Periodograms for Plavchan and BLS algorithms are for correlations with strongest L-S peaks. 
4-8 GHz mean quiescent flux density of $26.7 \pm 3.1 \mu \mathrm{Jy}$. Assuming an upper $3 \sigma_{\text {rms }}$ detection limit of $5.1 \mu \mathrm{Jy}$ for flux density averaged over $8-12 \mathrm{GHz}$, the upper limit spectral index is $\alpha \lesssim-3.2 \pm 0.7$ and the corresponding mildly relativistic power-law electron distribution index is $\delta \gtrsim 5.0$. For $2 \mathrm{M} 1043$, Kao et al. (2016) measured a $4-8 \mathrm{GHz}$ mean quiescent flux density of $16.3 \pm 2.5 \mu \mathrm{Jy}$, which leads to $\alpha \lesssim-3.0 \pm 0.7$ and $\delta \gtrsim 4.7$.

In the stellar case, typical spectral indices for quiescent radio emission from active $M$ dwarfs are much flatter at $\alpha \sim-0.3$ (e.g., Güdel et al. 1993; Güdel 1994, and references therein), although there may be fundamental differences for the brown dwarf case. While evidence exists that much of the quiescent emission from ultracool dwarfs exhibits behavior consistent with incoherent synchrotron or gyrosynchrotron emisssion (e.g., Ravi et al. 2011; Williams et al. 2015), there have been some objects that depart from this model.

At least some component of the "quiescent" non-pulsed emission may be coherent. The steep spectral index implied by the drop-off in quiescent emission is atypical but not impossible for nonthermal gyrosynchrotron or synchrotron emission (Dulk 1985; Melrose 2006) and may be more indicative of an emission cutoff. Such a model has been proposed for solar quiescent emission with electron power-law indices $\delta \approx 2-4$ and weak $\sim 100 \mathrm{G}$ fields (Pallavicini et al. 1985; White et al. 1989; White \& Franciosini 1995; Umana et al. 1998), including for both plasma and gyrosynchrotron emission.

Evidence for a coherent mechanism at play in the quiescent component precedes the data presented here. For instance, the L3.5 dwarf 2MASS J00361617+1821104 exhibits broadly varying emission with duty cycles $\sim 30 \%$ of the rotational period (Berger 2002; Hallinan et al. 2008). This emission can be decomposed into two components: (1) a periodic and highly circularly polarized component, which Hallinan et al. (2008) attributed to ECM, and (2) a component that was largely unpolarized for two out of three of the observed rotation periods. In the third rotation period, this second component emitted two narrower peaks with up to $\sim 75 \%$ right- and leftcircular polarization, respectively. This same feature was observed in data separated by 18 months, which demonstrated the longevity of this high degree of circular polarization and ruled out incoherent gyrosynchrotron as a mechanism. To explain the observed short-term variability in the degree of polarization, Hallinan et al. (2008) argued that local conditions in the emitting region could plausibly depolarize the emission, a phenomenon that commonly occurs in the strongly circularly polarized millisecond spikes of solar radio emission, such that polarization fractions can range from $0 \%$ to $10 \%$ (Benz 1986).

Similar dual-component varying emission has been observed in the T6 dwarf WISEP J112254.73+255021.5. The first component comprises clear bursts in left-circular polarization Route \& Wolszczan (2016). The second component is broadly varying in both the right- and left-circularly polarized flux density, with spectral index $\alpha=-1.5 \pm 0.3$ and a high degree of circular polarization $(>50 \%)$ that is present for nearly the entire duration of a 162-minute observation (Williams et al. 2017). This second component is similar to what we observed in SIMP0136 and 2M1237. These two objects have flatter spectra than SDSS0423 and 2M1043 if no variability is assumed, with spectral indices $\alpha \approx-2.1 \pm 0.4$ and $\alpha \approx-0.9 \pm 0.3$, respectively.
In the case that the non-pulsed emission is coherent, plasma emission is unlikely because the plasma density in a cool brown dwarf such as SDSS0423 is expected to be tenuous in comparison to the solar corona, and the plasma frequency scales with the electron density as $\nu_{p} \propto n_{e}^{1 / 2}$. For a gas to exhibit plasma-like behaviors, electron-electron interactions should dominate electron-neutral interactions. In models of thermal ionization for temperatures characteristic of $\mathrm{M}-\mathrm{T}$ dwarfs, Rodriguez-Barrera et al. (2015) find that while $\mathrm{M}$ dwarfs can expect $\sim 10^{-1}$ fraction of ionization in their atmospheres, this rapidly drops to $\sim 10^{-4}-10^{-3}$ for $1000 \mathrm{~K}$ objects. Additionally, the presence of plasma would correlate with X-ray emission, but L and later brown dwarfs remain underluminous in X-ray compared to their warmer counterparts (Williams et al. 2014). The other plausible coherent mechanism would be ECM emission in the form of superposed flares, as observed for 2MASS J00361617+1821104 (Hallinan et al. 2008). However, if the mechanism generating this quiescent emission is indeed related to the pulsed emission, the presence of the pulses observed in the same frequency bands would preclude the observed cutoff, unless the emitting regions traced different magnetic field strengths. This scenario could account for the strong circular polarization of the non-flaring emission from SIMP0136, 2M1237, and WISEP J112254.73+255021.5.

Another possible explanation is that the quiescent emission may exhibit long-term variability. Such variability has been previously reported in other brown dwarfs. For instance, Antonova et al. (2007) did not detect any radio emission from a $9 \mathrm{hr}$ observation (with $3 \sigma$ upper limit $\sim 45 \mu \mathrm{Jy}$ ) of 2MASS J05233822-1403022 (L2.5) on 2006 September 23, which Berger et al. (2010) also reported for observations on 2008 December 30. Archival data analyzed by Antonova et al. (2007) revealed that this same object was also not detected on 2004 May 03 with a $3 \sigma$ upper limit of $42 \mu \mathrm{Jy}$, yet it was detected without the flare on 2004 May 17 with a flux density of $95 \pm 19 \mu \mathrm{Jy}$ and also on 2004 June 18 with a flux density of $230 \pm 17 \mu \mathrm{Jy}$, the latter of which was previously reported by Berger (2006). Similarly, Berger et al. (2010) reported no detectable emission from BRI 0021 (M9.5) with $3 \sigma$ upper limits of $54 \mu \mathrm{Jy}$ and $48 \mu \mathrm{Jy}$ for $4.9 \mathrm{GHz}$ and $8.5 \mathrm{GHz}$, despite a previous marginal detection of its quiescent emission at $40 \pm 13 \mu \mathrm{Jy}$ as well as a flare with a peak flux density of $360 \pm 70 \mu \mathrm{Jy}$. In the case that the quiescent emission is variable over longer timescales, long-term monitoring of radio brown dwarfs would be necessary to quantify how much the current detection rate underestimates the true detection rate and may warrant revisiting previously undetected objects with $\mathrm{H} \alpha$ or infrared variability such as SDSS J12545393-0122474 (Kao et al. 2016).

The radio emission from $2 \mathrm{M} 1047$ differs from both the strongly polarized two-component behavior observed from SIMP0136 and 2M1237 and the single-component (pulsing only) behavior of SDSS0423 and 2M1043. Like SIMP0136 and 2M1237, the non-pulsing radio emission from 2M1047 is also relatively flat. Kao et al. 2016 measured a $4-8 \mathrm{GHz}$ mean quiescent flux density of $17.5 \pm 3.6 \mu \mathrm{Jy}$, implying $\alpha \approx-0.9 \pm 0.4$ and $\delta \approx 2.4$, when we take the $12-18 \mathrm{GHz}$ mean quiescent flux density. This confirms the spectral indices measured by Williams et al (2015) at $4-8 \mathrm{GHz}$ $(\alpha=0.0 \pm 0.3)$ and $8-12 \mathrm{GHz}(\alpha=-0.7 \pm 0.7)$. However, unlike SIMP0136 and 2M1237, the non-pulsing $12-18 \mathrm{GHz}$ emission from $2 \mathrm{M} 1047$ is not circularly polarized, and 


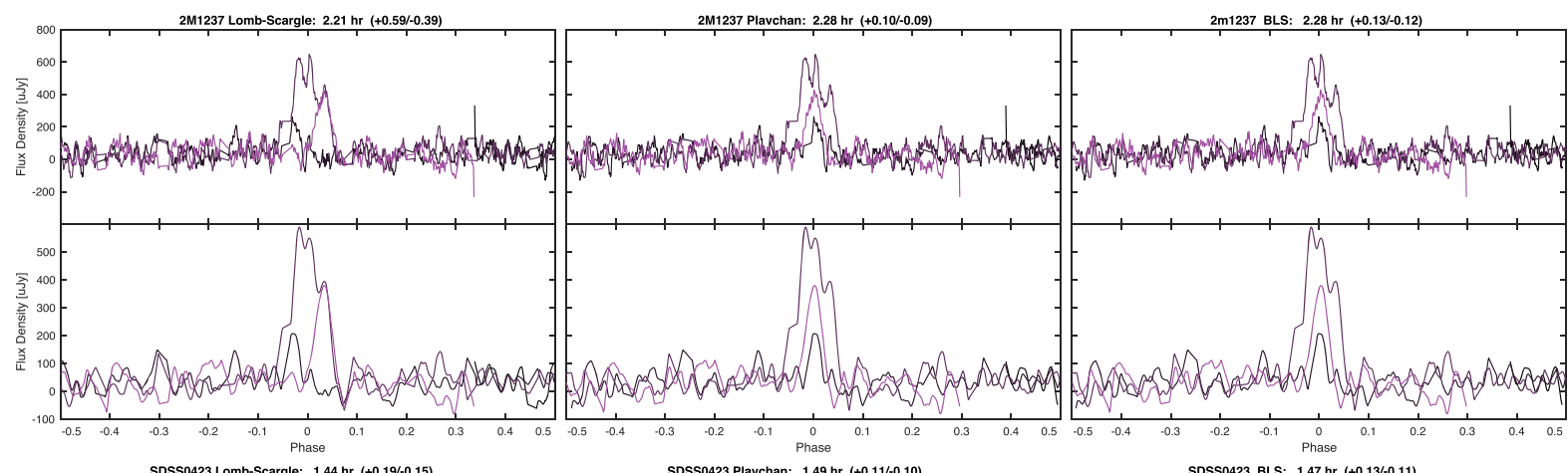

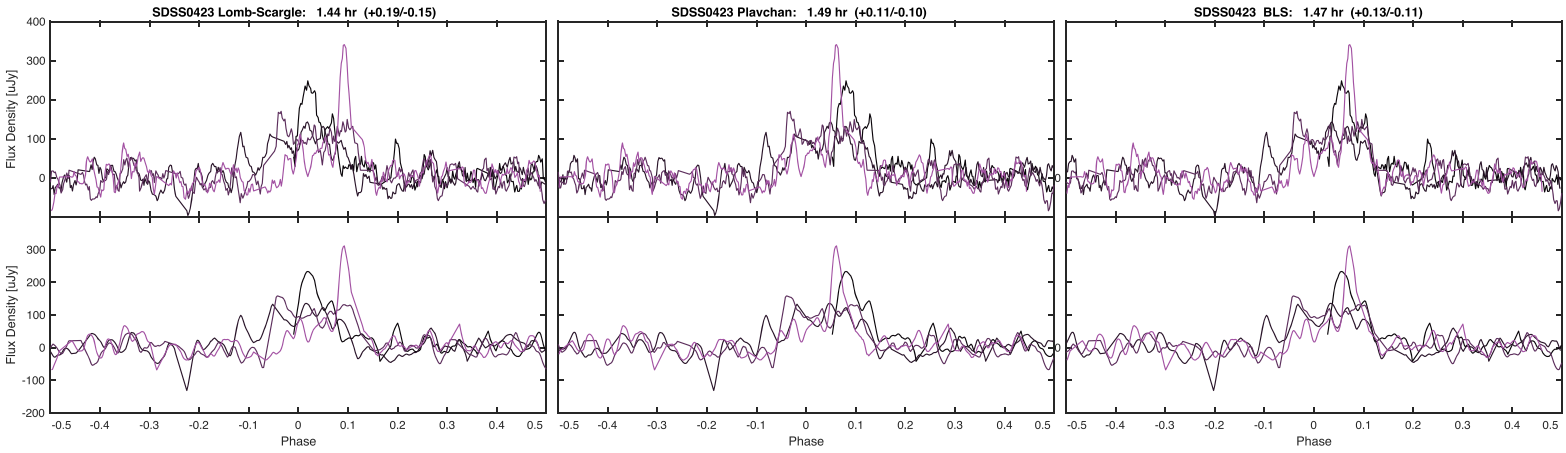
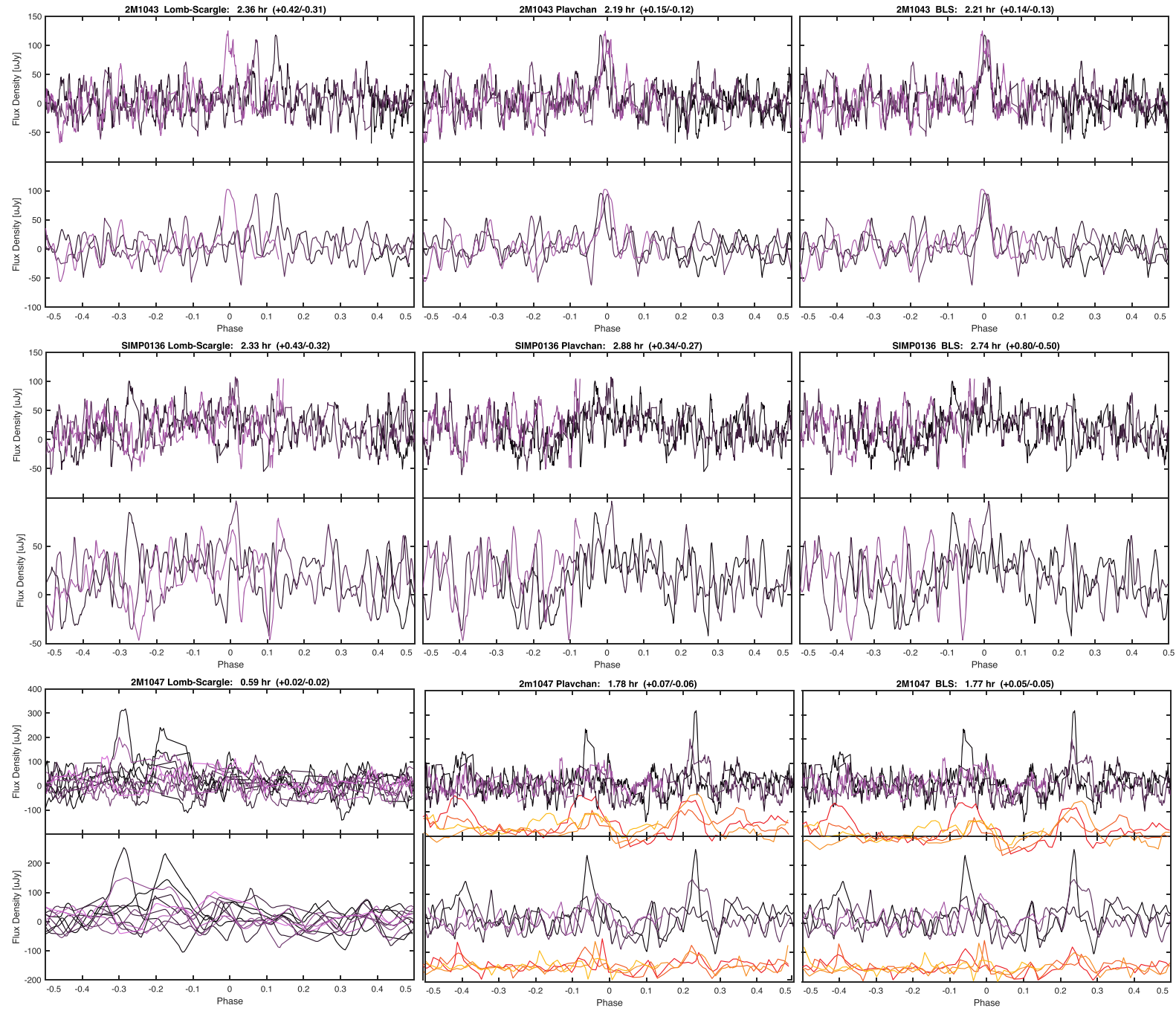

Figure 6. From left to right: Phase-folded $10 \mathrm{~s}$ time series using periods from L-S, Plavchan, and BLS periodograms. Top panels are raw data, bottom panels are smoothed data. $60 \mathrm{~s}$ time series are overplotted in orange. 


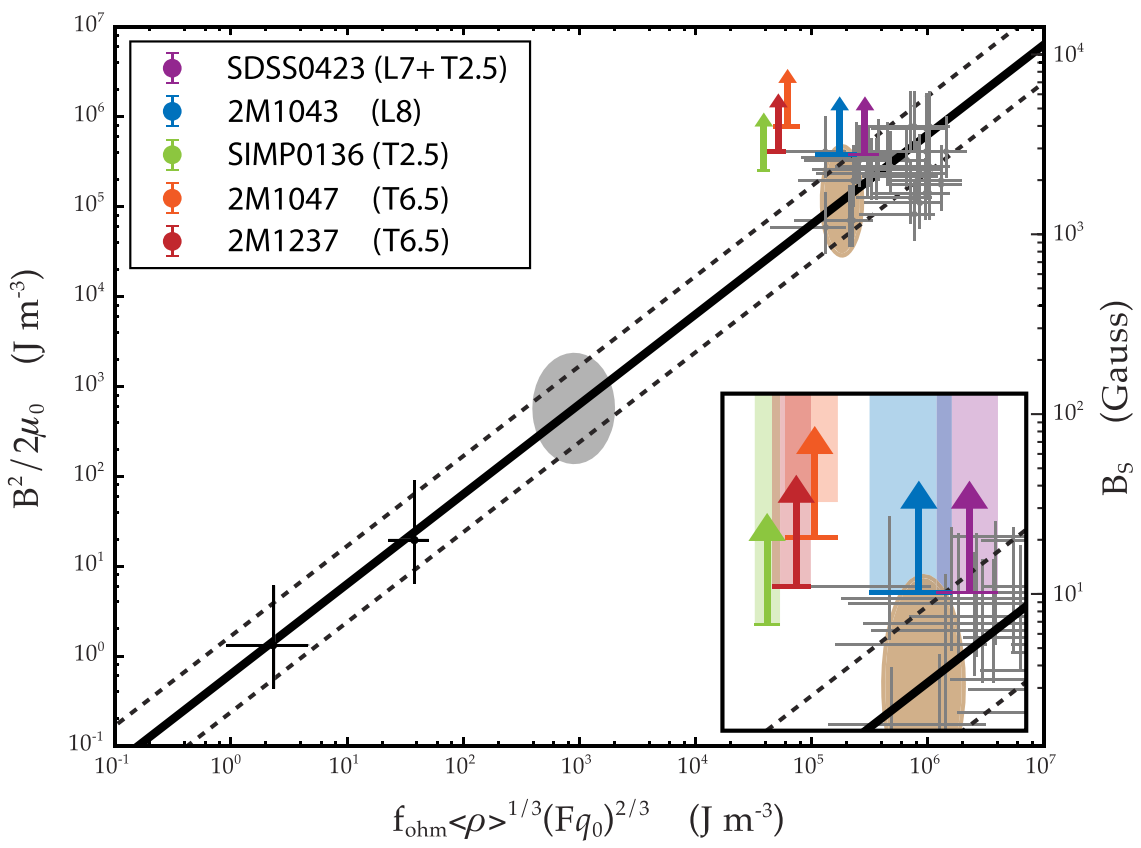

Figure 7. Comparison of estimated lower-bound magnetic field energy densities for our targets (overplotted arrows) to values predicted by the Christensen et al. (2009) scaling relation (black solid line) between convected energy density ( $x$-axis, $q_{0}$ ) and magnetic energy density (left $y$-axis) for fully convective dipole-dominated rapid rotators. Black dashed lines are $3 \sigma$ uncertainties on the scaling relationship, and horizontal bars on arrows are our estimated uncertainties. Previous constraints were T Tauri stars and old M dwarfs (gray crosses). Black points represent Earth and Jupiter. Brown and gray ellipses are predicted positions for a $1500 \mathrm{~K}$ brown dwarf and a $7 M_{\mathrm{J}}$ exoplanet, respectively. Right $y$-axis values are predicted surface-averaged fields $B_{s}$. Observed T dwarf magnetic fields depart from predicted magnetic energy densities.

Williams et al (2015) reported "quasi-quiescent" emission from $2 \mathrm{M} 1047$ at $4-8 \mathrm{GHz}$ that was not circularly polarized.

\subsection{Intermittent Pulses: Implications for ECM Emission Frequency Cutoff}

At these high frequencies, pulses appear to be more intermittent compared to previous 4-8 GHz observations, with short-duration variability in both time and frequency. For instance, while the central pulse in $2 \mathrm{M} 1237$ is present at all bandwidths, the right-most peak is clearly apparent only at 11-12 GHz. SDSS0423 emits two faint right-circularly polarized pulses at $8-9 \mathrm{GHz}$, but the right pulse appears to drop out at higher frequencies. For 2M1047, the multi-peaked and/or long-lived left-circularly polarized pulse at $12.8-13.5 \mathrm{GHz}$ early in the observing block drops out at higher frequencies, while three fainter left-circularly polarized pulses emerge at $15-16 \mathrm{GHz}$. In contrast, these objects' C-band (4-8 GHz) pulses are present at all sub-bands (Kao et al. 2016).

This pulse variability suggests that the conditions for current systems driving these auroral emissions may be much less stable or more variable close to the surface of the star, where fields are expected to be stronger and emitting frequencies are higher. One possibility for variable conditions is magnetic flux. While large-scale fields appear necessary to drive solar system auroral currents and the same may occur in isolated brown dwarfs like our targets, evolving and complex small-scale fields may also begin to emerge near the object surface. As radiating electrons traverse the large-scale field lines inward, they will radiate at higher frequencies corresponding to the increased magnetic fluxes that they see. Some fully convective dynamo models capable of generating kilogauss fields suggest that these small-scale fields may be driven by convection near the surface, where convective turnover times are shorter and small-scale intermittent features begin to appear in convective flows. In contrast, more stable large-scale fields may be tied to slowly overturning convection in the deep interiors (Browning 2008).

Other examples of intermittent auroral pulse structures exist in the literature. As an example, the dynamic spectrum of LSR J1835+3259 shows one pulse per rotation extending through $\sim 4-8 \mathrm{GHz}$, one extending through $\sim 4-6 \mathrm{GHz}$, and one only extending through $\sim 4.5 \mathrm{GHz}$, with emission from each pulse appearing to fade away or renew again at different frequencies (Hallinan et al. 2015). Narrowband and intermittent pulses have also been observed in terrestrial, Jovian, and Saturnian auroral kilometric radiation (AKR). Highresolution dynamic spectra reveal that rather than one continuous pulse through frequency, AKR actually consists of many small-scale micropulses from individually radiating sources that are highly time variable and narrowly spaced in frequency, with widths of the order of $\sim 10-1000 \mathrm{~Hz}$ corresponding to bunched groups of these local AKR sources traveling very rapidly through space. The origin of this fine structure remains unknown, but it is speculated that they may reflect a number of physical processes, including propagation and absorption effects or small-scale field parallel current structures (Gurnett et al. 1981; Pottelette et al. 1999; Treumann 2006, and references therein).

While we do observe what appears to be the disappearance of highly circularly polarized pulsed auroral emission in SIMP0136, 2M1043, and SDSS0423, in light of the observed behavior in 2M1237 and 2M1047 and the above-discussed cases, we classify these dropoffs only as very tentative evidence of ECM emission cutoff. The known intermittent behavior of AKR suggests that observations through a much wider bandwidth of high frequencies are necessary to confirm a true emission cutoff. 


\subsection{Comparison to Luminosity-driven Model}

Previously, Kao et al. (2016) found tentative evidence of a $\mathrm{T}$ dwarf departure from a predominantly luminosity-driven dynamo for rapid rotators $(P<4$ days). This dynamo scaling relationship extended planetary dynamo models to stellarmass objects including $T$ Tauri stars and old $M$ dwarfs, whose Zeeman broadening and ZDI measurements were empirically consistent with a scaling relationship linking internal magnetic energy density to convected energy flux and dynamo region density, while being largely independent of both magnetic diffusivity and rotation rate (Christensen et al. 2009, hereafter C09). The broad span through planetary and stellar parameter spaces suggested that the scaling law may in fact present a unifying principle governing the magnetic field generation in all rapidly rotating, dipoledominated, fully convective objects-namely, that the bolometric flux $q_{0}$ sets the magnetic field strength averaged over the whole volume of the dynamo region $\left\langle B^{2}\right\rangle$, with a weak dependence on the mean density of the dynamo region $\langle\rho\rangle$ :

$$
\left\langle B^{2}\right\rangle \propto\langle\rho\rangle^{1 / 3} q_{0}^{2 / 3}
$$

Because the C09 model is specific to dipole-dominated fields ( $>35 \%$ of the magnetic energy density in the dipole component) in rapid rotators, possible explanations for the observed tentative inconsistency between late- $\mathrm{L}$ and $\mathrm{T}$ dwarf magnetic fields with the C09 model included (1) higher-order non-dipole fields may dominate our objects, or (2) several of our targets may be slower rotators.

Regarding the possibility that our objects may not have dipole-dominated field topologies, auroral radio emission by itself is currently insufficient for confirming magnetic field topologies. This is because the frequency of the emission corresponds only to localized emitting regions in the magnetospheres of our targets. Therefore, in this work we make no attempt to assume a particular magnetic field topology and instead follow the formalism presented in Kao et al. (2016) to convert the local magnetic fields measured with ECM emission $B_{\mathrm{ECM}}$ into lower-bound mean surface field magnitudes $B_{\mathrm{s} \text {, dip }}$, which we list in Table 10. For this conversion, we conservatively adopt ECM emission cutoff frequencies corresponding to the middle of the last sub-band with imaging detections of auroral pulses in Stokes $I$ and $V$, since evidence of cutoffs in the ECM emission frequency is inconclusive (Section 6.2). As described in Kao et al. (2016), $B_{\mathrm{s} \text {, dip }}$ is equivalent to a lower-bound Zeeman broadening measurement of a surface-averaged field strength $B_{s}$, and the presence of any higher-order fields would raise this estimate. We convert $B_{\mathrm{s} \text {, dip }}$ into a mean internal field strength $\langle B\rangle$ for comparison to the C09 relation by following the conversions outlined in C09 and summarized in Kao et al. (2016).

It is important to note that although the Kao et al. (2016) formalism assumes that a dipole field powers the ECM emission, the lower-bound mean surface magnetic field calculated from this assumption accommodates multipolar field topologies. Therefore, in adopting this formalism to interpret our measured magnetic fields, we are not making a concrete statement on brown dwarf field topologies. However, the Kao et al. (2016) formalism depends on the observed and modeled magnetic behavior of fully convective $\mathrm{M}$ dwarfs extending to late-L and $\mathrm{T}$ dwarfs. We believe that an analogy to fully convective $\mathrm{M}$ dwarfs is appropriate here, as the dynamo action occurring in fully convective $\mathrm{M}$ dwarfs is likely similar to what occurs in very cold brown dwarfs. This is because the dynamo regions of $\sim \mathrm{M} 4$ and later dwarfs, including $\mathrm{L}$ and $\mathrm{T}$ dwarfs, are expected to be fully convective, with an important exception that we discuss below. In contrast, higher mass stars have dynamo region structures where both convection and differential rotation are important to the fluid dynamics driving the dynamos.

The differing fluid dynamics in these dynamo regions lead to different magnetic field behaviors. Strong differential rotation in the dynamo region tends to destroy the dominant dipolar component generated by convection (Gastine et al. 2012; Jones 2014), leading to toroidal magnetic fields (e.g., Browning 2008; Gastine et al. 2012; Yadav et al. 2016) and magnetic cycles (Yadav et al. 2016). While many late-type M dwarfs and brown dwarfs are expected to have fully convective dynamo regions, differential rotation may be able to arise in some. Observational evidence suggests that low-mass $M$ dwarfs may be able to generate magnetic fields that undergo cycles, pointing to dynamo mechanisms that may be solar-like (Wright \& Drake 2016). However, the onset of differential rotation suggested by such magnetic cycles seems to occur only in slowly rotating objects (Browning 2008; Yadav et al. 2016). Hence, for our assumption that the dynamo mechanism in fully convective $\mathrm{M}$ dwarfs is analogous to those in late- $\mathrm{L}$ and $\mathrm{T}$ dwarfs to hold, our objects must be rapid rotators.

Regarding the rapid-rotation requirement for both the C09 model and our use of the Kao et al. (2016) formalism, the periodicities that we recover in Section 4.3 together with cloud variability studies for SIMP0136 and C-band observations for 2M1047 by Williams \& Berger (2015) unambiguously confirm that our targets are indeed rapid rotators, with rotation periods between $\sim 1.44$ and $2.28 \mathrm{hr}$. While SIMP0136 does not have any clearly periodic pulse structure, infrared cloud variability studies suggest a rotation period of $2.3895 \pm 0.0005 \mathrm{hr}$ (Artigau et al. 2009; Croll et al. 2016). This rotation period is not inconsistent with the recovered periodicity in its quasiquiescent emission, which we measure to be $2.88_{-0.27}^{+0.34} \mathrm{hr}$. Our data confirm that to date, all pulsing radio brown dwarfs with rotation period measurements have reported rotational periods shorter than $4 \mathrm{hr}$ (Pineda et al. 2017, and references therein). These rotation periods likely fall well within the limit of rapid rotation (Rossby number Ro $<0.1$ ), with measured rotation periods of the order of just a few hours compared to convective turnover times that may be in the tens to hundreds of days (e.g., Noyes et al. 1984; Pizzolato et al. 2003; Landin et al. 2010; McLean et al. 2012).

The previous statement comes with some important caveats. First, empirical estimations and numerical calculations of convective turnover times with observable properties such as X-ray luminosity do not extend to L and T dwarfs. Second, dynamo regions can span a wide range of fluid densities, with density stratification ranging from $\sim 20 \%$ in incompressible fluids such as in the geodynamo to at least $\sim 10^{6}-10^{10}$ in stars and likely also cool brown dwarfs (Saumon et al. 1995). In highly stratified regimes, fluids in the most diffuse regions become less efficient at transporting heat, and small-scale motions with accompanying shorter convective turnover times may become increasingly important. Defining an appropriate Rossby number is not straightforward, since it is unclear where in the dynamo region is most important for generating fields that auroral radio emission probes. 
We present our resulting field constraints on a reproduction of the C09 scaling law in Figure 7, with $x$-axis values determined from the physical parameters of our targets summarized in Section 2. The T dwarfs 2M1047, 2M1237, and SIMP0136 clearly depart by an order of magnitude from the C09 magnetic energy predictions. While the late-L dwarfs lie near the outer bounds of the $3 \sigma$ error on the scaling relationship, these are in fact conservative constraints; no emission frequency cutoff has been conclusively detected, pointing to the possibility of yet stronger fields. This tantalizingly hints at a possible ultracool brown dwarf locus that may not age along the predicted luminosity-magnetic field sequence (Reiners \& Christensen 2010). Additional studies identifying aurorally pulsing radio brown dwarfs and characterizing their physical parameters could reveal such a locus.

As we previously pointed out, this emerging departure from the C09 predictions may be due to magnetic topologies that are not dominated by dipoles. In such a case, a comparison to the C09 predictions would be inappropriate, since the models employed by $\mathrm{C} 09$ are specific to objects with dipole-dominated fields. Here, we examine that possibility. While several attempts to model brown dwarf ECM emission with dipole fields have been successful (Kuznetsov et al. 2012; Nichols et al. 2012; Leto et al. 2016), Lynch et al. (2015) found that a dipole field was unable to reproduce observed dynamic spectra for the M9 dwarf TVLM 513-46546 and L0+L1.5 binary 2MASS J0746425+200032. Instead, ECM sources at the footprints of coronal magnetic loops with radial extents of only $1.2-2.7 R_{s}$, where $R_{s}$ is the dwarf radius, are more able to reproduce the observed emission, suggesting that a global multipolar field may be responsible for powering ECM emission in some cool dwarfs. It is plausible that the magnetic fields of these cool dwarfs may be dominated by small-scale multipolar fields, since dynamo models suggest that objects with very low Rossby numbers $(<0.1)$ can have magnetic topologies with most of the magnetic energy either in the dipole component or in multipolar components (Gastine et al. 2013), and ZDI studies show that dwarfs with kilogauss dipoles have order-of-magnitude weaker multipole fields and vice versa (Morin et al. 2010).

The radio pulsing of some dwarfs, including TVLM 51346546, the M8.5 dwarf LSR J1835+3259, and our targets here, is stable for many months and even years. The long-term persistence of these ECM pulses requires continuous quasistable particle acceleration. In the coronal loop model explored by Lynch et al. (2015), this would require reconnection in an active region that persists for many months, such as a planetary field continuously interacting with the dwarf field (Lanza 2013). In this scenario, the radio emission would be seen near the footprints of a sequence of coronal loops.

Quasi-stable particle acceleration can also be explained by an auroral model (Hallinan et al. 2015). If the emission that we observe from brown dwarfs is indeed auroral in nature, evidence points to a strong dipole field rather than a strong multipolar field. Auroral emissions rely on coupling energy from locations where there is a large $v \times B$ into the magnetosphere (Nichols et al. 2012). This is best achieved by having strong magnetic fields far away from the planet (e.g., in the middle or outer magnetosphere), where rotational speed $v$ will be high as well. Dipoles drop off much more slowly than higher-order fields and are more likely to dominate auroral power for this reason, suggesting that ECM emission of auroral
Table 10

Adopted Magnetic Fields

\begin{tabular}{lccc}
\hline \hline Object & $\begin{array}{c}\text { Tentative } \\
\nu_{\text {cutoff }}^{\mathrm{a}} \\
(\mathrm{GHz})\end{array}$ & $\begin{array}{c}\text { Local Field } \\
B_{\mathrm{ECM}}^{\mathrm{b}} \\
(\mathrm{kG})\end{array}$ & $\begin{array}{c}\text { Min avg Field } \\
B_{\text {s,dip }} \\
(\mathrm{kG})\end{array}$ \\
\hline 2M1047 & 15.75 & 5.6 & 4.0 \\
SIMP0136 & 9.0 & 3.2 & 2.3 \\
2M1043 & 11.0 & 3.9 & 2.8 \\
2M1237 & 11.5 & 4.1 & 2.9 \\
SDSS0423 & 11.0 & 3.9 & 2.8 \\
\hline
\end{tabular}

Notes.

${ }^{a}$ Center of highest sub-band with non-tentative imaging detection of ECM pulse.

${ }^{b} B_{\mathrm{ECM}[\mathrm{kG}]}=\nu_{\mathrm{ECM}[\mathrm{GHz}]} / 2.8$ (Treumann 2006).

c $\left\langle B_{\mathrm{s}, \mathrm{dip}}^{2}\right\rangle=\frac{1}{2} B_{\mathrm{ECM}}^{2}$ (Kao et al. 2016).

origin likely probes the dipole components of our objects. Indeed, all of the examples of planetary aurorae in our solar system demonstrate that the ECM emission can originate from a dipole component of our targets' magnetic fields. Similarly, models of the corotation breakdown mechanism that occurs in the Jovian auroral system assuming dipolar magnetic fields show close agreement between modeled and observed auroral radio luminosities for TVLM 513-46546 (M9), LSR J1835 +3259 (M8.5), and 2MASS J00361617+1821104 (L3.5) (Nichols et al. 2012; Turnpenney et al. 2017). This model also predicted rotation periods between $\sim 2.1$ and $2.8 \mathrm{hr}$ for 2M1047, which is not inconsistent with the rotation period measured by Williams \& Berger (2015).

\subsection{Consideration of Age-related Models}

The possibility that magnetic energy may scale with luminosity in rapidly rotating convective objects supports a picture in which brown dwarf magnetic fields are expected to decay with age as they cool through the $\mathrm{L} / \mathrm{T} / \mathrm{Y}$ spectral sequence and become increasingly less luminous. Indeed, field strengths can wane by a factor of 10 over the lifetime of a brown dwarf when evolutionary tracks are applied to the C09 model (Reiners \& Christensen 2010).

The luminosity of a brown dwarf depends both on its age and its mass, and these factors may account for some of the possible emerging disagreement between the C09 relationship and our targets. Using the Baraffe et al. (2003) brown dwarf evolutionary tracks and the C09 relationship, we calculated predicted age-evolving magnetic energy densities for each mass grid point and overplotted our objects in Figure 8. Given the disagreement between our objects and the $\mathrm{C} 09$ relation, it is no surprise that our objects also depart from these age-related predictions. However, while our $\mathrm{T}$ dwarf data appeared to disagree somewhat with the C09 model in Section 6.3, a departure was less clear for our L dwarfs. Accounting for the effects of age and mass on luminosity hints at a stronger departure from the C09 scaling law for our warmer but less massive and younger $\mathrm{L}$ dwarfs than was initially evident when mass and age were folded into luminosity. Regardless, a much larger sample is needed before any concrete conclusions can be drawn about how age affects convective dynamos, and the simplest prediction to test is whether objects with similar masses have stronger fields when younger. 


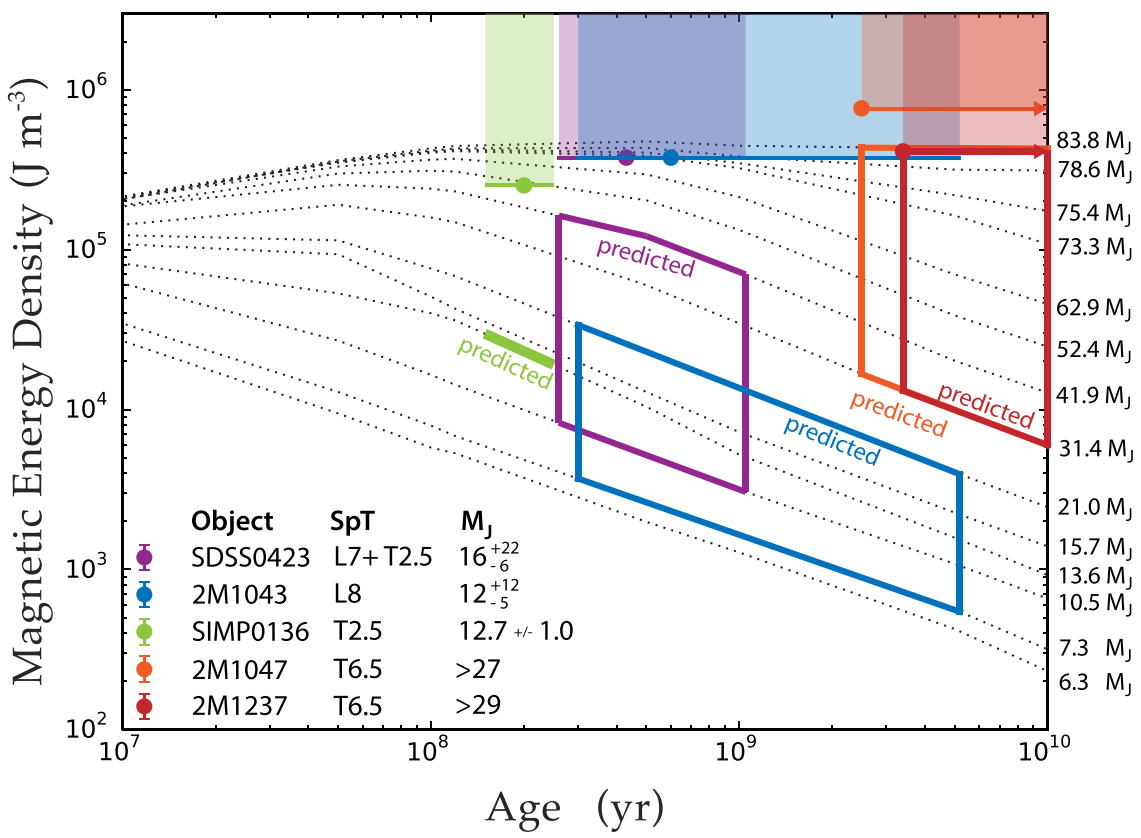

Figure 8. When effective temperature, age, and mass are accounted for by applying the Christensen et al. (2009) scaling relationship to Baraffe et al. (2003) brown dwarf evolutionary tracks, they are not sufficient to explain the strong magnetic fields generated by the dynamo mechanisms in our targets. Here, we compare inferred lower-bound magnetic field energy densities (colored circles) to predictions from dynamo evolutions tracks (outlined colored regions). Masses are adopted from Kao et al. (2016) and Gagné et al. (2017) and are provided in the bottom left corner. Outlined colored regions include mass tracks falling within the mass uncertainties or those nearest the edge cases. For objects with only lower-bound constraints on masses, we adopt $0.08 M_{\odot}$ as the nominal hydrogen-burning limit. Shaded colored regions depict age uncertainties and extend upward to indicate that magnetic energy densities inferred from auroral radio magnetic field measurements are lower bounds. Age constraints for 2M1047 and 2M1237 give only lower bounds.

In the event that luminosity $\left(T_{\text {eff }}\right)$ does not play a dominant role in brown dwarf dynamos, it is worth noting that magnetic field strengths do not appear to vary much by age across an order of magnitude between $\sim 0.2$ and 3.4 Gyr. Of course, no definitive ECM emission cutoff frequency has been observed for any brown dwarfs yet, including our targets, so the plotted mean surface field strengths are merely lower bounds and the future addition of constraints from higher frequencies and a broader range of ages, masses, and temperatures may yet reveal a correlation between age and field strength.

Presenting our data within the context of age has an important implication for ongoing efforts to detect exoplanet radio emission. While such efforts have focused on hot Jupiters (which see high flux from host stars, thus increasing the luminosity of solar-wind-generated aurorae) and hot young exoplanets (Lazio \& Farrell 2007; Lazio et al. 2010; Hallinan et al. 2013; Murphy et al. 2015; Lynch et al. 2017), old objects appear to also be capable of generating strong fields along with the associated radio emission, and broader searches may be warranted.

\subsection{First Radio Detection of a Planetary-mass Object?}

Recently, Gagné et al. (2017) reported that SIMP0136 may be a member of the $\sim 200$ Myr old Carina-Near moving group based on its kinematics, with a field interloper probability of only $0.0001 \%$. Using an empirical measurement of its bolometric luminosity and the Saumon \& Marley (2008) models, they inferred $R=1.22 \pm 0.01 R_{\mathrm{J}}$, which together predicted $T_{\text {eff }}=$ $1098 \pm 6 \mathrm{~K}$ and $M=12.7 \pm 1.0 M_{\mathrm{J}}$.

This low mass is further supported by new $v \sin i$ measurements that, in combination with its photometric periodicity, constrains its inclination angle at $i=55.9_{-1}^{+1}{ }^{\circ}$. . This inclination angle leads to a lower-bound radius and upper bounds on age and mass of
$R>1.01 \pm 0.02 R_{\mathrm{J}}, \tau<910_{-110}^{+26}$ Myr and $M<42.6_{-2.4}^{+2.5} M_{\mathrm{J}}$. Finally, models of the photometric variability assuming a single spot are also in agreement, constraining its inclination at $i<60^{\circ}$, which would increase the lower bound radius to $R>1.17 \pm 0.02 R_{\mathrm{J}}$, and further support the young age and low mass derived for SIMP0136 if it is indeed a member of the Carina-Near moving group.

This low mass of SIMP0136 is notable for its proximity to the $\sim 12-13 M_{\mathrm{J}}$ deuterium burning limit, or the mass above which compact gaseous objects are expected to burn deuterium (spiegel et al 2011; molliere \& Mordasini 2012; bodenheimer et al 2013). The deuterium burning limit is one way to distinguish between gas giant planets and brown dwarfs.

\section{Conclusions}

We detected auroral radio emission from four L7-T6.5 dwarfs up through 10-12 GHz, and one T6.5 object up through $15-16.5 \mathrm{GHz}$, corresponding to $3.2-5.6 \mathrm{kG}$ local magnetic field strengths and 2.3-4.0 kG minimum surface-averaged fields. Additionally, we reported a tentative $16.5-18 \mathrm{GHz}$ auroral pulse detection for the T6.5 dwarf 2M1047, corresponding to $6.2 \mathrm{kG}$ local magnetic field strengths and $4.4 \mathrm{kG}$ minimum surface-averaged fields. Pulses appear to be more intermittent in frequency at higher frequencies compared to previous observations of lower frequency counterparts, which can be interpreted as evidence of a higher degree of variability in the conditions necessary to generate auroral radio emission near the surfaces of brown dwarfs. While we observe the fading-out of auroral pulses at $11-12 \mathrm{GHz}$ for some targets, observations at higher frequencies are necessary to affirm definitive cutoffs in the auroral radio emission. We additionally observe no detectable quiescent emission for SDSS0423 and 2M1043, but do observe highly circularly polarized non-pulsed emission 
from SIMP0136 and in some sub-bands also for 2M1237. The behavior of SDSS0423 and 2M1043 may point to long-term variability in the quiescent emission mechanism, while SIMP0136 and 2M1237 are more suggestive of coherent processes.

The presented detections are strong direct constraints on dynamo theory at the substellar-planetary boundary. We presented data suggesting that a scaling relation between convected energy flux and magnetic energy density (Christensen et al. 2009) may not fit. We also show that age, mass, and temperature together cannot account for the strong magnetic fields produced by our targets. Using the rotational modulation of auroral radio emission, we measured rotational periods between 1.47 and $2.28 \mathrm{hr}$ for SDSS0423, 2M1043, and 2M1237. These short rotation periods are consistent with periods measured for earlier-type brown dwarfs using auroral radio emission, and they reiterate that rapid rotators can host strong large-scale fields. Finally, we find that our oldest targets $(>2.5 \mathrm{Gyr})$ can generate fields that are as strong as those measured in our youngest targets ( 200-600 Myr), suggesting that old exoplanets may also host fields with strengths comparable to their younger siblings and serving as preliminary and very tentative evidence that age dependence in dynamo mechanisms may be weak. The absence of an emission frequency cutoff means that we have not broken any degeneracies in our analyses and that a larger, more well-characterized sample is required.

Included in our sample was the archetypal cloud variable SIMP0136, which was recently found to be a member of a nearby $\sim 200$ Myr moving group. This new age constraint reduces its estimated mass to a mere $12.7 \pm 1.0 M_{\mathrm{J}}$, possibly making SIMP0136 the first known planetary-mass object detected in the radio. If SIMP0136 is indeed a field exoplanet, its detection demonstrates that auroral radio emission can open a new avenue to detecting exoplanets, including elusive rogue planets.

M.M.K. thanks Jackie Villadsen for helping to troubleshoot calibrations and Rakesh Yadav for thoughtful and instructive discussions about dynamo modeling. M.M.K. additionally thanks the enthusiastically supportive staff at the National Radio Astronomy Observatory for their technical mentorship.

Support for this work was provided by the NSF through the Grote Reber Fellowship Program administered by Associated Universities, Inc./National Radio Astronomy Observatory. The National Radio Astronomy Observatory is a facility of the National Science Foundation operated under cooperative agreement by Associated Universities, Inc.

This material is based in part upon work supported by the National Science Foundation under Grant AST-1654815 and the NASA Solar System Exploration Virtual Institute cooperative agreement 80ARC017M0006. G.H. acknowledges the support of the Alfred P. Sloan Foundation and the Research Corporation for Science Advancement.

J.S.P. was supported by a grant from the National Science Foundation Graduate Research Fellowship under grant no. DGE-1144469.

This publication makes use of data products from the Two Micron All Sky Survey, which is a joint project of the University of Massachusetts and the Infrared Processing and Analysis Center/California Institute of Technology, funded by the National Aeronautics and Space Administration and the National Science Foundation.
Facility: VLA.

Software: CASA (McMullin et al. 2007), MATLAB (MATLAB Signal Processing Toolbox, R2016a).

\section{ORCID iDs}

Melodie M. Kao (iD https://orcid.org/0000-0001-5125-1414 J. Sebastian Pineda (iD https://orcid.org/0000-0002-4489-0135 David Stevenson (1) https://orcid.org/0000-0001-9432-7159 Adam Burgasser (ib https://orcid.org/0000-0002-6523-9536

\section{References}

Ackerman, A. S., \& Marley, M. S. 2001, ApJ, 556, 872

Antonova, A., Doyle, J. G., Hallinan, G., Golden, A., \& Koen, C. 2007, A\&A, 472,257

Antonova, A., Hallinan, G., Doyle, J. G., et al. 2013, A\&A, 549, A131

Apai, D., Radigan, J., Buenzli, E., et al. 2013, ApJ, 768, 121

Artigau, É., Bouchard, S., Doyon, R., \& Lafrenière, D. 2009, ApJ, 701, 1534

Artigau, É., Doyon, R., Lafrenière, D., et al. 2006, ApJL, 651, L57

Artigau, E., Nadeau, D., \& Doyon, R. 2003, in IAU Symp. 211, Brown Dwarfs, ed. E. Martin (San Francisco, CA: ASP), 451

Badman, S. V., Branduardi-Raymont, G., Galand, M., et al. 2015, SSRv, 187, 99

Baraffe, I., Chabrier, G., Barman, T. S., Allard, F., \& Hauschildt, P. H. 2003, A\&A, 402, 701

Batygin, K., \& Stevenson, D. J. 2010, ApJL, 714, L238

Benz, A. O. 1986, SoPh, 104, 99

Berdyugina, S. V., \& Solanki, S. K. 2002, A\&A, 385, 701

Berger, E. 2002, ApJ, 572, 503

Berger, E. 2006, ApJ, 648, 629

Berger, E., Ball, S., Becker, K. M., et al. 2001, Natur, 410, 338

Berger, E., Basri, G., Fleming, T. A., et al. 2010, ApJ, 709, 332

Berger, E., Rutledge, R. E., Phan-Bao, N., et al. 2009, ApJ, 695, 310

Berger, E., Rutledge, R. E., Reid, I. N., et al. 2005, ApJ, 627, 960

Brain, D. A., McFadden, J. P., Halekas, J. S., et al. 2015, GeoRL, 42, 9142

Browning, M. K. 2008, ApJ, 676, 1262

Bodenheimer, P., D’Angelo, G., Lissauer, J. J., et al. 2013, ApJ, 770, 120

Burgasser, A. J. 2007, ApJ, 659, 655

Burgasser, A. J., Burrows, A., \& Kirkpatrick, J. D. 2006a, ApJ, 639, 1095

Burgasser, A. J., Geballe, T. R., Leggett, S. K., Kirkpatrick, J. D., \& Golimowski, D. A. 2006b, ApJ, 637, 1067

Burgasser, A. J., Kirkpatrick, J. D., Brown, M. E., et al. 1999, ApJL, 522, L65

Burgasser, A. J., Kirkpatrick, J. D., Liebert, J., \& Burrows, A. 2003, ApJ, 594, 510

Burgasser, A. J., Kirkpatrick, J. D., Reid, I. N., et al. 2000, AJ, 120, 473

Burgasser, A. J., Liebert, J., Kirkpatrick, J. D., \& Gizis, J. E. 2002a, AJ, 123,2744

Burgasser, A. J., Marley, M. S., Ackerman, A. S., et al. 2002b, ApJL, 571, L151

Burgasser, A. J., Reid, I. N., Leggett, S. K., et al. 2005, ApJL, 634, L177

Carson, J. C., Marengo, M., Patten, B. M., et al. 2011, ApJ, 743, 141

Chabrier, G., \& Küker, M. 2006, A\&A, 446, 1027

Christensen, U. R., \& Aubert, J. 2006, GeoJI, 166, 97

Christensen, U. R., Holzwarth, V., \& Reiners, A. 2009, Natur, 457, 167

Clarke, F. J., Hodgkin, S. T., Oppenheimer, B. R., Robertson, J., \& Haubois, X. 2008, MNRAS, 386, 2009

Croll, B., Muirhead, P. S., Lichtman, J., et al. 2016, arXiv:1609.03587

Cruz, K. L., Reid, I. N., Kirkpatrick, J. D., et al. 2007, AJ, 133, 439

Cruz, K. L., Reid, I. N., Liebert, J., Kirkpatrick, J. D., \& Lowrance, P. J. 2003, AJ, 126, 2421

Donati, J.-F., Forveille, T., Collier Cameron, A., et al. 2006, Sci, 311, 633

Dulk, G. A. 1985, ARA\&A, 23, 169

Dupuy, T. J., \& Liu, M. C. 2017, ApJS, 231, 15

Enoch, M. L., Brown, M. E., \& Burgasser, A. J. 2003, AJ, 126, 1006

Gagné, J., Faherty, J. K., Burgasser, A. J., et al. 2017, ApJL, 841, L1

Gastine, T., Duarte, L., \& Wicht, J. 2012, A\&A, 546, A19

Gastine, T., Morin, J., Duarte, L., et al. 2013, A\&A, 549, L5

Geballe, T. R., Knapp, G. R., Leggett, S. K., et al. 2002, ApJ, 564, 466

Güdel, M. 1994, ApJS, 90, 743

Güdel, M. 2002, ARA\&A, 40, 217

Güdel, M., Schmitt, J. H. M. M., Bookbinder, J. A., \& Fleming, T. A. 1993, ApJ, 415, 236

Gurnett, D. A., Kurth, W. S., \& Scarf, F. L. 1981, Natur, 292, 733 
Hallinan, G., Antonova, A., Doyle, J. G., et al. 2006, ApJ, 653, 690 Hallinan, G., Antonova, A., Doyle, J. G., et al. 2008, ApJ, 684, 644 Hallinan, G., Bourke, S., Lane, C., et al. 2007, ApJL, 663, L25 Hallinan, G., Littlefair, S. P., Cotter, G., et al. 2015, Natur, 523, 568 Hallinan, G., Sirothia, S. K., Antonova, A., et al. 2013, ApJ, 762, 34 Hartmann, L., Herczeg, G., \& Calvet, N. 2016, ARA\&A, 54, 135 Johns-Krull, C. M., \& Valenti, J. A. 1996, ApJL, 459, L95

Johns-Krull, C. M., \& Valenti, J. A. 2000, in ASP Conf. Ser. 198, Stellar Clusters and Associations: Convection, Rotation, and Dynamos, ed. R. Pallavicini, G. Micela, \& S. Sciortino (San Francisco, CA: ASP), 371 Jones, C. A. 2014, Icar, 241, 148

Kao, M. M., Hallinan, G., Pineda, J. S., et al. 2016, ApJ, 818, 24

Kervella, P., Mérand, A., Ledoux, C., Demory, B.-O., \& Le Bouquin, J.-B. 2016, A\&A, 593, A127

Kirkpatrick, J. D., Cruz, K. L., Barman, T. S., et al. 2008, ApJ, 689, 1295

Kovács, G., Zucker, S., \& Mazeh, T. 2002, A\&A, 391, 369

Kuznetsov, A. A., Doyle, J. G., Yu, S., et al. 2012, ApJ, 746, 99

Landin, N. R., Mendes, L. T. S., \& Vaz, L. P. R. 2010, A\&A, 510, A46

Lanza, A. F. 2013, A\&A, 557, A31

Lazio, T. J., Carmichael, S., Clark, J., et al. 2010, AJ, 139, 96

Lazio, T. J., \& Farrell, W. M. 2007, ApJ, 668, 1182

Leblanc, F., Modolo, R., Curry, S., et al. 2015, GeoRL, 42, 9135

Leto, P., Trigilio, C., Buemi, C. S., et al. 2016, MNRAS, 459, 1159

Lynch, C., Mutel, R. L., \& Güdel, M. 2015, ApJ, 802, 106

Lynch, C. R., Murphy, T., Kaplan, D. L., Ireland, M., \& Bell, M. E. 2017, MNRAS, 467, 3447

Marley, M. S., Saumon, D., \& Goldblatt, C. 2010, ApJL, 723, L117

MATLAB Signal Processing Toolbox, R2016a MATLAB Signal Processing Toolbox (Natick, MA: The MathWorks Inc.)

McLean, M., Berger, E., \& Reiners, A. 2012, ApJ, 746, 23

McMullin, J. P., Waters, B., Schiebel, D., Young, W., \& Golap, K. 2007, in ASP Conf. Ser. 376, Astronomical Data Analysis Software and Systems XVI, ed. R. A. Shaw, F. Hill, \& D. J. Bell (San Francisco, CA: ASP), 127

Melrose, D. B. 2006, ApJ, 637, 1113

Miles-Páez, P. A., Metchev, S. A., Heinze, A., \& Apai, D. 2017, ApJ, 840, 83

Mohanty, S., \& Basri, G. 2003, ApJ, 583, 451

Mohanty, S., Basri, G., Shu, F., Allard, F., \& Chabrier, G. 2002, ApJ, 571, 469

Mollière, P., \& Mordasini, C. 2012, A\&A, 547, A105

Morin, J., Donati, J.-F., Petit, P., et al. 2010, MNRAS, 407, 2269

Murphy, T., Bell, M. E., Kaplan, D. L., et al. 2015, MNRAS, 446, 2560

Nichols, J. D., Burleigh, M. R., Casewell, S. L., et al. 2012, ApJ, 760, 59

Noyes, R. W., Hartmann, L. W., Baliunas, S. L., Duncan, D. K., \& Vaughan, A. H. 1984, ApJ, 279, 763

Pallavicini, R., Willson, R. F., \& Lang, K. R. 1985, A\&A, 149, 95

Parks, J. R., Plavchan, P., White, R. J., \& Gee, A. H. 2014, ApJS, 211, 3

Perley, R. A., \& Butler, B. J. 2013, ApJS, 204, 19

Pineda, J. S., Hallinan, G., \& Kao, M. M. 2017, ApJ, 846, 75

Pineda, J. S., Hallinan, G., Kirkpatrick, J. D., et al. 2016, ApJ, 826, 73

Pizzolato, N., Maggio, A., Micela, G., Sciortino, S., \& Ventura, P. 2003, A\&A, 397, 147

Plavchan, P., Jura, M., Kirkpatrick, J. D., Cutri, R. M., \& Gallagher, S. C. 2008, ApJS, 175, 191
Pottelette, R., Ergun, R. E., Treumann, R. A., et al. 1999, GeoRL, 26, 2629 Prato, L., Mace, G. N., Rice, E. L., et al. 2015, ApJ, 808, 12

Radigan, J. 2014, ApJ, 797, 120

Radigan, J., Lafrenière, D., Jayawardhana, R., \& Artigau, E. 2014, ApJ, 793, 75 Ravi, V., Hallinan, G., Hobbs, G., \& Champion, D. J. 2011, ApJL, 735, L2 Reiners, A. 2012, LRSP, 9, 1

Reiners, A., \& Basri, G. 2006, ApJ, 644, 497

Reiners, A., \& Basri, G. 2007, ApJ, 656, 1121

Reiners, A., \& Basri, G. 2008, ApJ, 684, 1390

Reiners, A., \& Basri, G. 2009, A\&A, 496, 787

Reiners, A., \& Basri, G. 2010, ApJ, 710, 924

Reiners, A., \& Christensen, U. R. 2010, A\&A, 522, A13

Rodriguez-Barrera, M. I., Helling, C., Stark, C. R., \& Rice, A. M. 2015, MNRAS, 454, 3977

Rosén, L., Kochukhov, O., \& Wade, G. A. 2015, ApJ, 805, 169

Route, M. 2016, ApJL, 830, L27

Route, M., \& Wolszczan, A. 2012, ApJL, 747, L22

Route, M., \& Wolszczan, A. 2016, ApJL, 821, L21

Saumon, D., Chabrier, G., \& van Horn, H. M. 1995, ApJS, 99, 713

Saumon, D., \& Marley, M. S. 2008, ApJ, 689, 1327

Schmidt, S. J., Hawley, S. L., West, A. A., et al. 2015, AJ, 149, 158

Schmidt, S. J., West, A. A., Hawley, S. L., \& Pineda, J. S. 2010, AJ, 139, 1808

Schmitt, J. H. M. M., \& Rosso, C. 1988, A\&A, 191, 99

Shulyak, D., Reiners, A., Engeln, A., et al. 2017, NatAs, 1, 0184

Shulyak, D., Reiners, A., Wende, S., et al. 2010, A\&A, 523, A37

Skrutskie, M. F., Cutri, R. M., Stiening, R., et al. 2006, AJ, 131, 1163

Spiegel, D. S., Burrows, A., Milsom, J. A., et al. 2011, ApJ, 727, 57

Stellingwerf, R. F. 1978, ApJ, 224, 953

Treumann, R. A. 2006, A\&ARv, 13, 229

Turnpenney, S., Nichols, J. D., Wynn, G. A., \& Casewell, S. L. 2017, MNRAS, 470, 4274

Ulmschneider, P. 2003, in Lectures on Solar Physics, Vol. 619, ed H. M. Antia, A. Bhatnagar, \& P. Ulmschneider (Berlin: Springer), 232 Umana, G., Trigilio, C., \& Catalano, S. 1998, A\&A, 329, 1010

Valenti, J. A., Marcy, G. W., \& Basri, G. 1995, ApJ, 439, 939

Vernazza, J. E., Avrett, E. H., \& Loeser, R. 1981, ApJS, 45, 635

Vidotto, A. A., Jardine, M., Morin, J., et al. 2013, A\&A, 557, A67

Vrba, F. J., Henden, A. A., Luginbuhl, C. B., et al. 2004, AJ, 127, 2948

Weinberger, A. J., Boss, A. P., Keiser, S. A., et al. 2016, AJ, 152, 24

White, S. M., \& Franciosini, E. 1995, ApJ, 444, 342

White, S. M., Kundu, M. R., \& Jackson, P. D. 1989, A\&A, 225, 112

Williams, P. K. G., \& Berger, E. 2015, ApJ, 808, 189

Williams, P. K. G., Berger, E., \& Zauderer, B. A. 2013, ApJL, 767, L30

Williams, P. K. G., Casewell, S. L., Stark, C. R., et al. 2015, ApJ, 815, 64

Williams, P. K. G., Cook, B. A., \& Berger, E. 2014, ApJ, 785, 9

Williams, P. K. G., Gizis, J. E., \& Berger, E. 2017, ApJ, 834, 117

Wilson, P. A., Rajan, A., \& Patience, J. 2014, A\&A, 566, A111

Wright, N. J., \& Drake, J. J. 2016, Natur, 535, 526

Yadav, R. K., Christensen, U. R., Morin, J., et al. 2015, ApJL, 813, L31

Yadav, R. K., Christensen, U. R., Wolk, S. J., \& Poppenhaeger, K. 2016, ApJL, 833, L28

Zarka, P. 1998, JGR, 103, 20159 